\title{
Two phase modeling of the influence of plastic strain on the magnetic and magnetostrictive behaviors of ferromagnetic materials
}

\author{
Olivier HUBERT ${ }^{\mathrm{a}, *}$, Said LAZREG ${ }^{\mathrm{a}}$ \\ ${ }^{a}$ LMT-Cachan (ENS-Cachan, UMR CNRS 8535, University Paris-Saclay) 61, avenue du président Wilson 94235 \\ Cachan cedex, France
}

\begin{abstract}
A growing interest of automotive industry in the use of high performance steels is observed. These materials are obtained thanks to complex manufacturing processes whose parameters fluctuations lead to strong variations of microstructure and mechanical properties. The on-line magnetic non-destructive monitoring is a relevant response to this problem but it requires fast models sensitive to different parameters of the forming process. The plastic deformation is one of these important parameters. Indeed, ferromagnetic materials are known to be sensitive to stress application and especially to plastic strains. In this paper, a macroscopic approach using the kinematic hardening is proposed to model this behavior, considering a plastic strained material as a two phase system. Relationship between kinematic hardening and residual stress is defined in this framework. Since stress fields are multiaxial, an uniaxial equivalent stress is calculated and introduced inside the so-called magneto-mechanical multidomain modeling to represent the effect of plastic strain. The modeling approach is complemented by many experiments involving magnetic and magnetostrictive measurements. They are carried out with or without applied stress, using a dual-phase steel deformed at different levels. The main interest of this material is that the mechanically hard phase, soft phase and the kinematic hardening can be clearly identified thanks to simple experiments. It is shown how this model can be extended to single phase materials.
\end{abstract}

Keywords: Magneto-mechanics, plastic straining, kinematic strengthening, magnetostriction, anhysteretic behavior, dual-phase steel, multiscale modeling

\section{Introduction}

Since the early works of Mateucci and Villari, mechanical stress has been known to significantly change the magnetic behavior of materials (see for instance the works reported in [1]) as well as their magnetostrictive behavior [2]. Many other works have shown that macroscopic magnetic behavior is sensitive to any mechanical loading depending on the loading level (elastic, plastic), the loading sign (tension, compression), and the loading nature (static or dynamic, uniaxial or multiaxial stress). The correlation between mechanical, metallurgical and magnetic

\footnotetext{
${ }^{*}$ Corresponding author

Email address: olivier.hubert@lmt.ens-cachan.fr (Olivier HUBERT) Preprint submitted to Journal of Magnetism and Magnetic Materials
} 
states has received increasing attention these last years due to the new ability and requirement to perform magnetic non-destructive monitoring (NDM) of materials and structures [3, 4, 5]. Steel manufacturers, for example, plan to generalize the implementation of in-situ magnetic NDM $[6,7,8]$ to control the mechanical and metallurgical state of high performance steels (dualphase, TRIP and TWIP steels [9, 11, 12,13]). The mechanical behavior of these steels is highly sensitive to the thermo-mechanical history of the material (heat treatments, rolling rate...) and especially sensitive to small variations in the process (e.g. furnace temperature) [9, 10]. For example, the small plastic strains experienced after a skin-pass of a dual-phase steel exhibiting a yield strength of about $450 \mathrm{MPa}$ (DP780) can be easily detected by a magnetic measurement.

Plastic strain leads to strong non linear changes in the magnetic behavior $[1,14,15,16,17]$. Experiments performed with various carbon steels [18, 19, 20, 21, 22, 23], electrical steels [16, $17,24,25]$, iron-cobalt [26, 27] or nickel alloys [1] have shown that the degradation occurs at the early stages of plastic strain [28, 29]. This change of magnetic behavior (usually qualified as a "degradation" since magnetic losses are increased and permeability decreased) is associated with a change of magnetostrictive behavior $[20,22,30,31]$ that can not be neglected in a general objective of understanding and modeling. Figure 1 illustrates, for instance, the strong change of magnetic and magnetostrictive behavior of a common iron-silicon electrical steel submitted to a very low plastic strain after a tensile test [30].

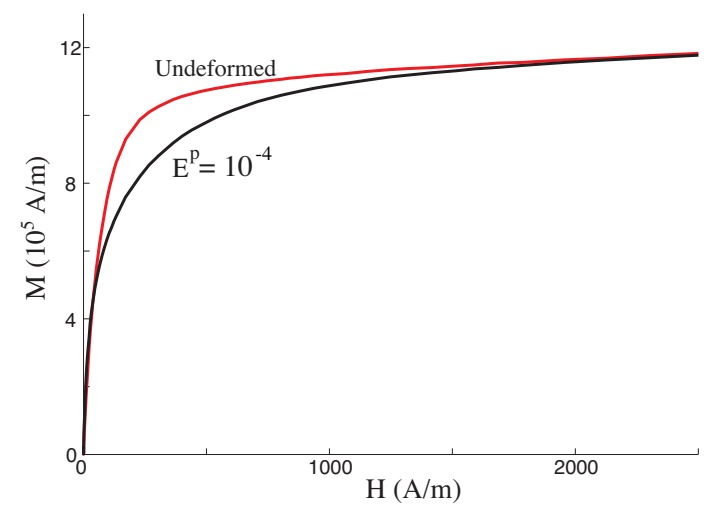

(a) magnetic behavior

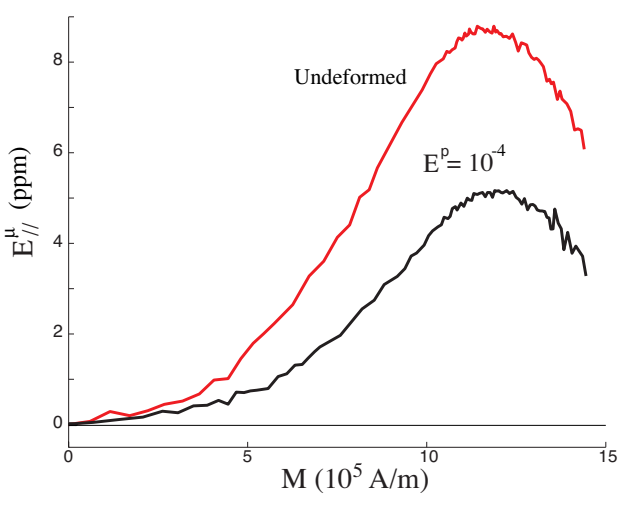

(b) magnetostrictive behavior

Figure 1: Influence of a very small plastic straining $(0.01 \%)$ on the anhysteretic magnetic and magnetostrictive behavior of non-oriented $3 \%$ silicon-iron [30] - quantities are defined in the next sections.

The influence of plastic deformation on the magnetic state has been studied by many authors, some of them interested by physical mechanisms at the local scale [32, 33, 14], the others looking at the influence of cutting (and associated plasticity) on the global response of an electrical machine $[34,35,36]$. Interactions between the magnetic microstructure (magnetic domains and walls) and the mechanical microstructure (dislocations, grains, stress fields) are the basis of the phenomenon.

The formulation of an accurate magneto-plastic coupling model provides a correlation between the plastic state and the magnetic behavior parameters. Several authors have tried to express the degradation of the magnetic state as a function of the dislocation density and pinning centers for domain walls $[37,38]$ and to integrate it into macroscopic approaches. Indeed the 
microstructural defect density increases significantly with the plastic deformation (isolated dislocations, dislocation tangles and walls,...). Some phenomenological models couple the magnetic behavior and plasticity via the dislocation density [39]. However, the evolution of the dislocation density is much more regular with the accumulated plastic strain than the evolution of magnetic properties $[16,17]$. To represent the plastic state, other authors have thought to use the configuration of dislocations $[40,41]$ by correlating the degradation of the magnetic state with the hardening of the material. This approach was then clarified in [17] where a link has been made between the magnetic state of a plastically deformed sample and the internal stress level. It is found that the plastic deformation is usually accompanied by a generation of internal stress. The effect of plasticity can be then interpreted as a heterogeneous strain effect, whose amplitude and wavelength depend on the microstructural configurations. This mechanical approach was not immediately retained in the scientific community. Most authors still prefer the phenomenological approaches making a link between the change of magnetic parameters (coercive field, magnetic susceptibility, core losses) to the plastic strain level [16, 42, 35, 29] or the stress level reached during deformation [21]. The consequence is that few of these models are able to propose a complete relationship between the stress path including multiaxial state and plastic deformation and the magnetic behavior.

A micro-macro modeling has been previously proposed to reach this goal. This model describes the influence of plastic strain on the overall magneto-mechanical behavior [25, 30]. It first involves a microcrystalline plasticity approach where the material is defined by its orientation distribution function (ODF). Since plastic flow is different from one grain to another due to different grain sizes and orientations, the stress field becomes heterogeneous and leads to residual stresses when the material is unloaded. The next step consisted in introducing the residual stress tensor solution of the first problem as a loading at the grain scale of a magnetic multiscale and multiaxial model able to describe magnetic and magnetostrictive behaviors [43]. This model was applied to non-oriented $\mathrm{Fe}-3 \% \mathrm{Si}$ and simulations of the effect of plasticity are consistent with experimental observations $[25,30]$. However, this approach was limited to the plastic deformation range corresponding to intergranular internal stresses. In addition, only the monotonic loading was taken into account and only at the unloaded state. The principle of considering the plasticity as an internal stress state is preserved in the new proposition presented in the paper. Lastly, the challenge is to simplify the micro-macro approach to reduce the computation time for potential NDM applications and make this approach more accessible to the magnetic materials community.

In this paper a macroscopic approach for the modeling of the influence of plastic deformation on the magnetic and magnetostrictive behaviors of ferromagnetic materials is proposed. The main assumption is that the material must be considered as a two phase material, with a mechanically "hard" phase and a mechanically "soft" phase (high and low yield strength) and appropriate volume fractions. The material is plastically strained leading to a residual stress field that can be related to the macroscopic quantity called kinematic hardening (or kinematic strenghtening, or backstress). Since stress fields are multiaxial, a magneto-mechanical equivalent stress criterion is applied to calculate the corresponding uniaxial mechanical loading [45]. The exact same two phases material is next considered for the magnetic modeling: the so-called multidomain modeling is applied to each phase as already done in [46] for Fe-Al-B alloys. Stresses calculated from the kinematic hardening and strain incompatibilities are used as loadings of the magnetic problem. The average modeling magnetic and magnetostrictive behaviors are finally obtained at a given level of plastic deformation. A superimposed macroscopic stress can be considered if 
appropriate.

This theoretical approach is complemented by several experiments involving magnetic and magnetostrictive measurements implemented using a DP780 steel where (mechanically) hard and soft phases are clearly defined. Results obtained using this material allow on the other hand to illustrate various points highlighted in the modeling section. Former experimental results are finally discussed in light of the proposed new approach.

\section{Magnetic and mechanical states, associated variables}

\subsection{Magnetic state and associated variables}

Magnetic materials are media which can be magnetized in presence of magnetic field. Their magnetic state can be described by the relationship between two vectors: the magnetic field $\vec{H}$ and the magnetization $\vec{M}$. The magnetic behavior is given by: $\vec{M}=\chi \vec{H}$ where $\chi$ is the second order magnetic susceptibility tensor. It depends non linearly on the magnetic field and $\vec{M}$ describes therefore a non-linear evolution as a function of $\vec{H}$. The norm of the magnetization reaches a saturation value noted $M_{s}$ which is function of the material composition.

Under an alternative magnetic field, the magnetization forms a hysteresis loop illustrating the irreversibility of the magnetic behavior and the presence of dissipative phenomena. This loop is usually characterized by the magnetic field at zero magnetization called coercive field $H_{c}$ and its area which defines the energy losses per cycle. Figure 2 illustrates the typical uniaxial $M(H)$ behavior of the DP780 steel. Anhysteretic and cyclic behaviors carried out at $\mathrm{f}=0.1 \mathrm{~Hz}$ are reported (see paragraph 4.3 for details about the experimental procedure).

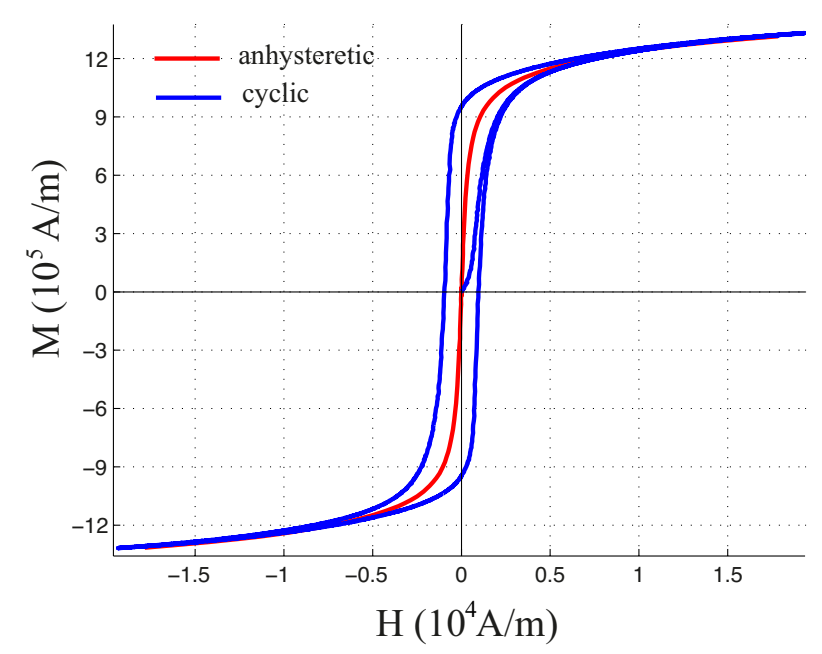

Figure 2: Magnetic behavior of DP780 steel - cyclic and anhysteretic behaviors.

The application of a magnetic field also induces a deformation of the material. The phenomenon is called magnetostriction and represented by the deformation tensor $\mathbf{E}^{\mu}$. It is a spontaneous intrinsic deformation of the material. It depends on the material's magnetic state and 
is representative of a local state coupling. The amplitude of deformation depends on many microstructural parameters such as the magnetic domains distribution and saturation magnetostriction $\left(\lambda_{100}\right.$ and $\lambda_{111}$ for a cubic symmetry material).

The evolution of the macroscopic deformation relative to the magnetic field $\mathbf{E}^{\mu}(\vec{H})$ (or associated magnetization $\vec{M}$ ) describes the magnetostrictive behavior of the material. This behavior is nonlinear, usually considered as irreversible, and sometimes non monotonic. The magnetostriction in the direction of applied field reaches a saturation value noted $\lambda_{s}$ which is a function of the material composition and the ODF. Figure 3 illustrates the typical evolution of magnetostriction measured in the direction of the applied field $\mathrm{E}_{/ /}^{\mu}(M)$ for the DP780 steel (with $\mathrm{E}_{/ /}^{\mu}={ }^{t} \vec{n} \cdot \mathbf{E}^{\mu} \cdot \vec{n}, \vec{n}$ denoting the direction of applied field). The anhysteretic behavior and cyclic behavior ${ }^{1}$ carried out at $\mathrm{f}=0.1 \mathrm{~Hz}$ are reported (see paragraph 4.3 for details about the experimental procedure).

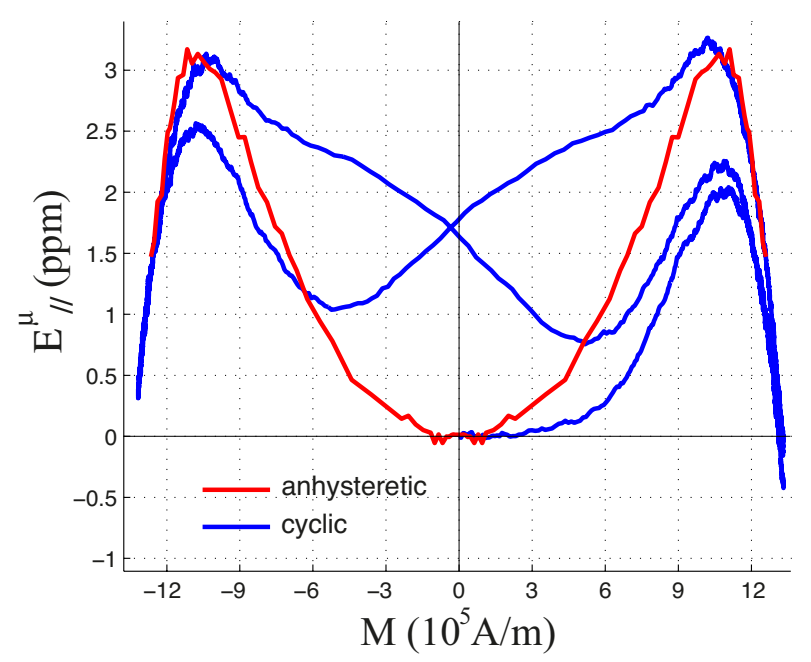

Figure 3: Magnetostrictive behavior of DP780 steel - cyclic and anhysteretic behaviors.

A plastic deformation usually strongly changes both magnetic and magnetostrictive behaviors as outlined in the introduction.

\subsection{Mechanical state and associated variables}

The mechanical behavior of a material is defined by its stress vs. strain response. Strain and stress are second order symmetric tensors denoted respectively $\mathbf{E}$ and $\boldsymbol{\Sigma}$. The elasto-plastic response is commonly defined by a mathematical relationship developed in plasticity theory. In the framework of linear elasticity stress and elastic strain are related by the constant fourth order stiffness tensor $\mathbb{C}$ so that:

$$
\boldsymbol{\Sigma}=\mathbb{C}: \mathbf{E}^{e}
$$

\footnotetext{
${ }^{1}$ The irreversible character of cyclic magnetostriction is mainly due to filtering effects (low-pass $10 \mathrm{~Hz}$ filtering for data plotted in Figure 3).
} 
The elastic limit (elastic-plastic transition) is defined by a yield surface in the stress space defined as a scalar function $f(\boldsymbol{\Sigma})=0$ depending on the criterion employed (equivalent stress $\Sigma_{e q}$ ) and the reference yield strength $\Sigma_{y}$ :

$$
f(\Sigma)=\Sigma_{e q}(\Sigma)-\Sigma_{y}
$$

The flow rules (expression of plastic straining $\mathbf{E}^{p}$ as function of the stress path) are usually the framework of associated plasticity for standard materials [47]. A normality hypothesis associated with the Hill principle leads to:

$$
\dot{\mathbf{E}}^{p}=\dot{\lambda} \frac{d f(\boldsymbol{\Sigma})}{d \mathbf{\Sigma}}
$$

where $\dot{\lambda}$ denotes the hardening parameter that can be related to the plastic strain tensor depending on the chosen yield function. An additivity hypothesis (valuable for small elastic deformation) leads to the calculation of the total deformation after time integration of equation (3):

$$
\mathbf{E}=\mathbf{E}^{e}+\mathbf{E}^{p}
$$

The $\boldsymbol{\Sigma}(\mathbf{E})$ relationship is illustrated in Figure 4 for the DP780 steel in the case of a tensile test (axial stress $\Sigma$ and longitudinal deformation $\mathrm{E}$ - rational representation). Complementary hardening rules allow one to describe a progressive change of the yield surface during plastic straining and the relationship between multiaxial stress and multiaxial plastic straining rates. The so-called isotropic hardening, $R$, gives the change of size of the yield surface; the kinematic hardening $\mathbf{X}$ gives the yield surface translation in the stress space.

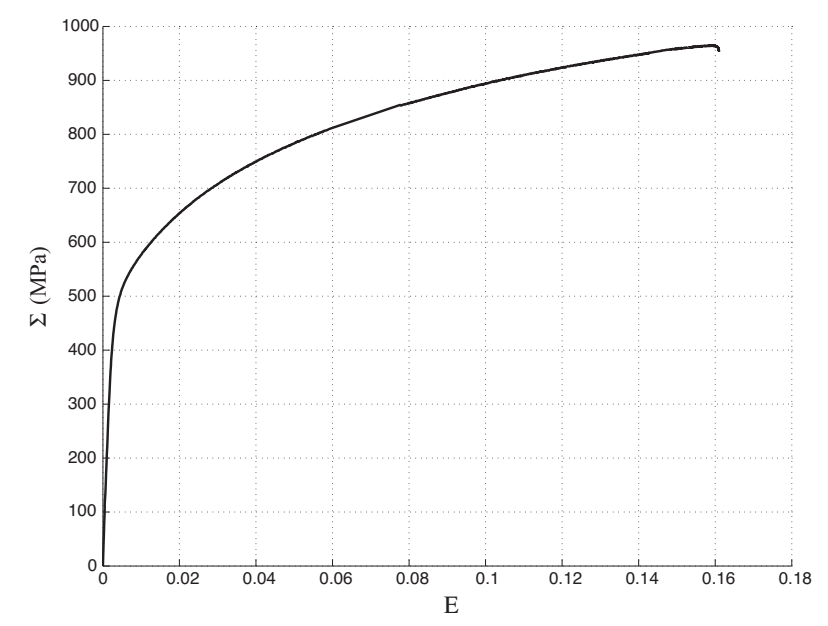

Figure 4: Tensile test of a DP780 steel - true stress / strain representation.

\subsection{Definition of kinematic hardening}

It is well established that, in most metallic materials, the stress/strain $\Sigma(\mathrm{E})$ tension- compression test shows a dissymmetry in the yield strength (figure 5). Indeed, the yield strength 
in compression is weaker than in tension after a tensile strengthening, leading to a shift of the elastic domain. This phenomenon is the Bauschinger effect. The same kind of dissymmetry is observed when loading begins by a compression. The Bauschinger effect can be directly related to the metallurgical state of the material. It is an indicator of the material heterogeneity: the more heterogeneous the structure, the stronger the Bauschinger effect. Heterogeneities in the material can be either intra-granular (dislocation tangles and walls) and/or inter-granular (strain incompatibilities) depending on the plastic strain level [48, 49]. The stress field consecutive to these configurations is usually called internal stress and the Bauschinger effect is its mechanical signature. Most metallic materials display this phenomenon.

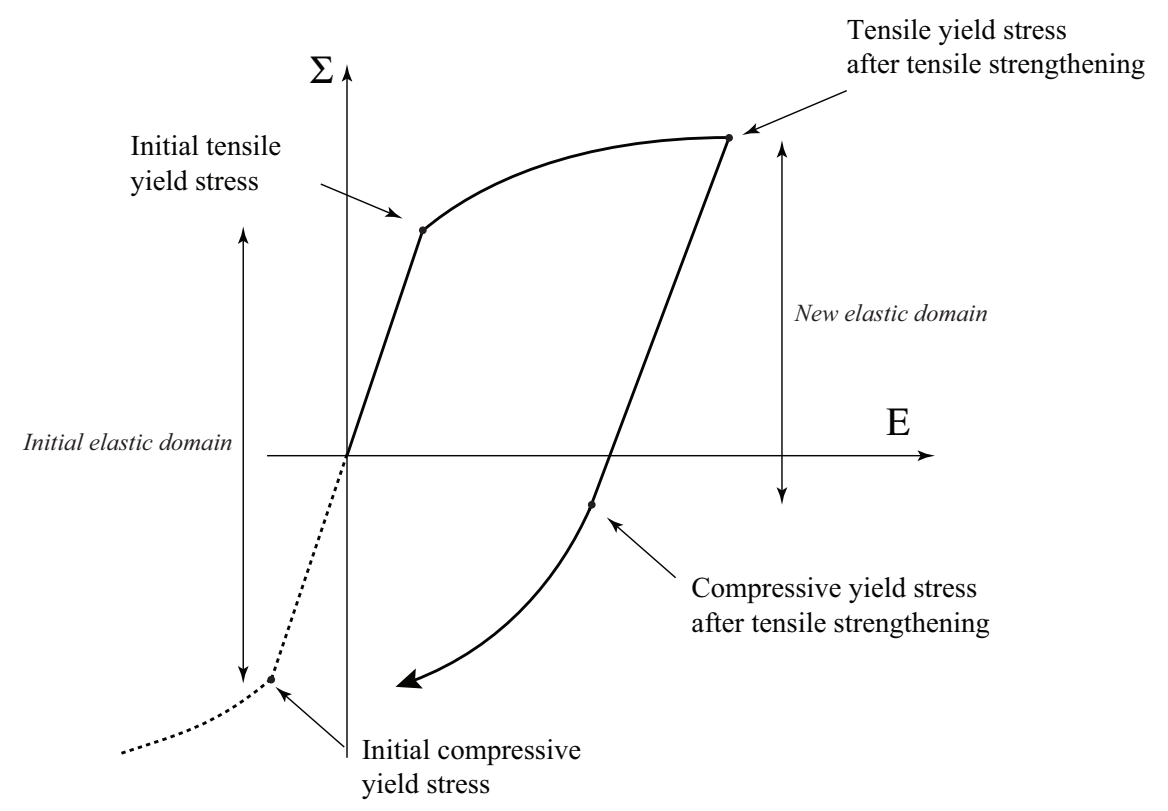

Figure 5: Uniaxial tension-compression test and illustration of the Bauschinger effect.

This effect is taken into account in plasticity rules via the kinematic hardening tensor $\mathbf{X}$ [47, 48]. Considering a von Mises criterion, an isotropic hardening R, a stress $\boldsymbol{\Sigma}$, and a yield strength $\Sigma_{y}$, the yield function is given by:

$$
f(\boldsymbol{\Sigma})=\sqrt{\frac{2}{3}(\mathbf{S}-\mathbf{X}):(\mathbf{S}-\mathbf{X})}-\mathbf{R}-\Sigma_{y}
$$

with

$$
\mathbf{S}=\boldsymbol{\Sigma}-\frac{1}{3} \operatorname{tr}(\boldsymbol{\Sigma}) \mathbf{I}
$$

the stress deviator.

$\mathbf{X}$ is the center of the yield surface in the deviatoric plane as illustrated in Figure 6. This tensor is deviatoric. The two usual flow rules that describe the kinematic stress rate $\dot{\mathbf{X}}$ as function of plastic strain rate are the linear Prager and the non-linear Armstrong and Frederick rules [47]. 


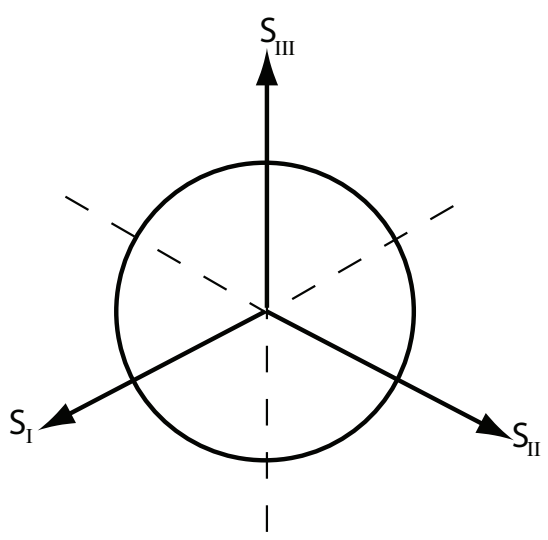

(a)

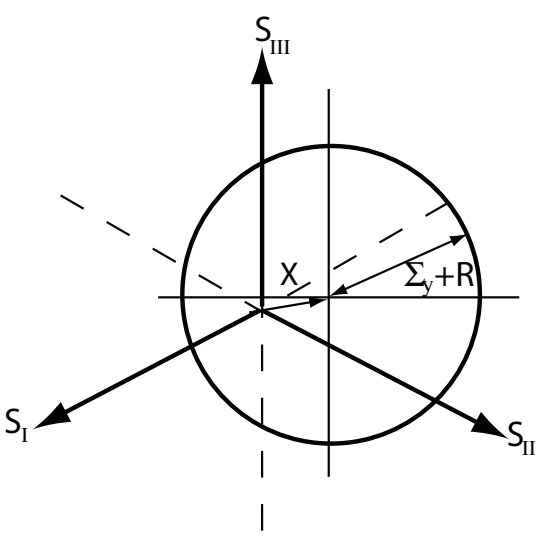

(b)

Figure 6: Translation of von Mises yielding surface associated to kinematic hardening $\mathbf{X}$ : (a) initial yield surface; (b) yield surface after strengthening.

We will suppose that the mechanical behavior of the materials considered for application of the modeling proposed in this paper are simple enough to be described by the mechanical variables detailed above.

\section{Macroscopic modeling of the influence of plastic straining on magnetic behavior}

\subsection{A previous multiscale modeling of the effect of plasticity}

The experimental studies reporting the effect of plastic straining on the magnetic behavior are numerous (few of them report the associated changes in the magnetostriction behavior). No satisfying model has been proposed except the micro-macro model associated with the multiscale magnetic model that is reviewed in this section. The only few existing macroscopic models are polynomial functions where parameters vary with the plastic strain level [35, 29]. Variation of parameters is usually strongly non linear with plastic strain level (or cumulative plasticity when a multiaxial loading is considered) and applicable for only one particular loading history. On the other hand, some works showed that plastic strains can be interpreted as internal stresses with a characteristic wavelength linked to the deformation level [17, 50]. Indeed several authors remarked that plastic straining and applied stress lead to comparable effects on the magnetic behavior [2, 51, 20, 21, 22]. The first assumptions and modeling approaches were made by Astié [40] for high purity iron and discussed in terms of kinematic hardening by Hubert [17] for a non-oriented 3\%silicon-iron alloy. The first numerical application used a multiscale approach $[25,30]$.

The multiscale modeling procedure uses sequently the calculation of local stress field and the multiscale magnetic modeling. Magnetization and magnetostriction behaviors are finally calculated. Reference [25] explains in details how the local plastic straining is calculated using microplasticity tools. References [43] and [44] explain in details the principle of multiscale modeling of magneto mechanical behavior. In this approach the effect of plastic straining on the magnetic and magnetostrictive behaviors is only explained by stress heterogeneities and associated magneto-elastic effects through the magneto-elastic energy term. No complementary 
parameter is introduced that makes this modeling the first physically based model able to describe the effect of plastic straining in a multiaxial framework.

Nonetheless the micro macro approach requires the precise knowledge of plastic microscopic parameters (grain size distribution, crystallographic orientations, hardening rules at the crystal scale), that makes this model not easy to extend to other materials. The micro-macro approach leads on the other hand to highly time consuming calculations which makes it irrelevant for a NDM procedure.

The new macroscopic approach proposed below uses a much more simple direct relationship between kinematic hardening and magnetic quantities.

\subsection{Mechanical modeling}

\subsubsection{General framework}

As detailed in the previous paragraph, plastic straining leads to multiaxial residual stresses at the grain scale. The multigrain plastic medium can be considered as a multiphased composite medium. The simplest description of such a medium is to consider a two phase medium, as initially proposed by Mughrabi [49], with a soft phase $s$ and a hard phase $h$, meaning that the $s$ phase exhibits a lower yield strength and strengthening than the $h$ phase. $f_{s}$ and $f_{h}$ indicate the volume fraction of $s$ and $h$ phases. The representative volume element (RVE) made of these two phases is submitted to stress tensor $\mathbf{\Sigma}$. $\mathbf{E}^{e}, \mathbf{E}^{p}$ and $\mathbf{E}$ denote the elastic, plastic and total strain tensors respectively so that :

$$
\mathbf{E}=\mathbf{E}^{e}+\mathbf{E}^{p}=\mathbb{C}^{-1}: \boldsymbol{\Sigma}+\mathbf{E}^{p}
$$

$\mathbb{C}$ indicates the stiffness tensor of the medium. The same decomposition can be made for each phase:

$$
\boldsymbol{\epsilon}_{h}=\boldsymbol{\epsilon}_{h}^{e}+\boldsymbol{\epsilon}_{h}^{p}=\mathbb{C}_{h}^{-1}: \boldsymbol{\sigma}_{h}+\boldsymbol{\epsilon}_{h}^{p} \quad \boldsymbol{\epsilon}_{s}=\boldsymbol{\epsilon}_{s}^{e}+\boldsymbol{\epsilon}_{s}^{p}=\mathbb{C}_{s}^{-1}: \boldsymbol{\sigma}_{s}+\boldsymbol{\epsilon}_{s}^{p}
$$

where $\mathbb{C}_{s}$ and $\mathbb{C}_{h}$ indicate the stiffness tensor of the soft and hard phases. Macroscopic stress and strain $\boldsymbol{\Sigma}$ and $\mathbf{E}$ always verify:

$$
\boldsymbol{\Sigma}=f_{h} \boldsymbol{\sigma}_{h}+f_{s} \boldsymbol{\sigma}_{s} \quad \mathbf{E}=f_{h} \boldsymbol{\epsilon}_{h}+f_{s} \boldsymbol{\epsilon}_{s}
$$

The local stress is given on the other hand by the Hill's relationship [53] so that:

$$
\boldsymbol{\sigma}_{s}=\boldsymbol{\Sigma}+\mathbb{C}^{*}:\left(\mathbf{E}-\boldsymbol{\epsilon}_{s}\right) \quad \sigma_{h}=\boldsymbol{\Sigma}+\mathbb{C}^{*}:\left(\mathbf{E}-\boldsymbol{\epsilon}_{h}\right)
$$

$\mathbb{C}^{*}$ indicates the Hill's constraint tensor. Strains are separated in elastic and plastic parts. After few calculations, the Berveiller-Zaoui relationship is obtained [54].

$$
\begin{aligned}
& \sigma_{s}=\mathbb{B}_{s}: \boldsymbol{\Sigma}+\mathbb{C}_{s}^{a c c}:\left(\mathbf{E}^{p}-\boldsymbol{\epsilon}_{s}^{p}\right) \\
& \boldsymbol{\sigma}_{h}=\mathbb{B}_{h}: \boldsymbol{\Sigma}+\mathbb{C}_{h}^{a c c}:\left(\mathbf{E}^{p}-\boldsymbol{\epsilon}_{h}^{p}\right)
\end{aligned}
$$

$\mathbb{C}_{s}^{a c c}$ and $\mathbb{C}_{h}^{a c c}$ are two fourth order accommodation stiffness tensors. $\mathbb{B}_{s}$ and $\mathbb{B}_{h}$ are two stress localization tensors depending on Hill's constraint, macroscopic and local (hard or soft) stiffness tensors. It is next possible to define two residual stress tensors $\mathbf{D}_{s}$ and $\mathbf{D}_{h}$ satisfying:

$$
\begin{aligned}
& \sigma_{s}=\mathbb{B}_{s}: \boldsymbol{\Sigma}+\mathbf{D}_{s} \\
& \sigma_{h}=\mathbb{B}_{h}: \boldsymbol{\Sigma}+\mathbf{D}_{h}
\end{aligned}
$$


and

$$
\begin{aligned}
f_{s} \mathbf{D}_{s}+f_{h} \mathbf{D}_{h} & =\mathbf{0} \\
f_{s} \mathbb{B}_{s}+f_{h} \mathbb{B}_{h} & =\mathbb{I}
\end{aligned}
$$

On the other hand an isotropic flow rule is considered for the hard and soft phases. Moreover a von Mises criterion is used for each phase. It is assumed though this description that the kinematic hardening is only linked to the interphase heterogeneities. Yield functions for both phases are:

$$
\begin{aligned}
& f\left(\sigma_{s}\right)=\sqrt{\frac{3}{2} \mathbf{s}_{s}: \mathbf{s}_{s}}-\sigma_{y s}-\mathbf{R}_{s} \\
& f\left(\sigma_{h}\right)=\sqrt{\frac{3}{2} \mathbf{s}_{h}: \mathbf{s}_{h}}-\sigma_{y h}-\mathbf{R}_{h}
\end{aligned}
$$

$\mathbf{s}_{i}, \sigma_{y i}$ and $\mathbf{R}_{i}$ are respectively the deviatoric tensor, yield strength and isotropic hardening of phase $i$.

The macroscopic yield function is expressed as a function of the macroscopic deviatoric stress tensor $\mathbf{S}$, yield strength $\Sigma_{y}$, isotropic $R$ and kinematic $\mathbf{X}$ hardening components using a von Mises criterion:

$$
f(\boldsymbol{\Sigma})=\sqrt{\frac{3}{2}(\mathbf{S}-\mathbf{X}):(\mathbf{S}-\mathbf{X})}-\Sigma_{y}-\mathbf{R}
$$

It should be noted that only the knowledge of the macroscopic stress state and the kinematic hardening is sufficient to define the stress state in each phase. This avoids a fastidious and imprecise estimation of the constitutive behavior of each phase, including localization operators and accommodation tensors.

\subsubsection{Virtual loading-unloading tensile test}

Figure 7 shows the different yield functions in the deviatoric plane for the particular case of $50 \%$ hard and 50\% soft phases. Due to the von Mises criterion, all yield functions are circles. The RVE composed of soft and hard phases without initial residual stress is submitted to a proportional loading along axis $(A)$ in the deviatoric plane. Due to isotropic hardening, the initial yield functions (blue and red plain lines) and final yield functions of both phases keep the same center at zero stress (point $O$ ). The initial yield function of the RVE corresponds to the initial yield function of the soft phase. The elastic domain increases in size due to isotropic hardening of the soft phase first, and isotropic hardening of the hard phase next, at higher stress level. After deformation of both phases, the stress point $A_{1}$ is reached, positioned at a distance between $h$ and $s$ phases yield functions depending on the ratio of the phases (equal distance in the present case). When unloaded, the stress in soft phase moves from point $B_{1}$ to point $B_{2}$ at the diametric opposite position in the circle. Because stress heterogeneity between phases and RVE remains constant in the elastic domain, the macroscopic yield function is delimited by point $A_{2}$ at the diametric opposite position of the corresponding circle. Deviatoric vectors $\underline{B_{1} A_{1}}$ and $\underline{\overrightarrow{B_{2} A_{2}}}$ are equal so that the center of macroscopic yield function after plastic straining is translated from point $O$ to point $O^{\prime}$. Deviatoric vectors verify:

$$
\overrightarrow{O^{\prime}}=\underline{B_{1} A_{1}}=\underline{\overrightarrow{B_{2} A_{2}}}=\mathbf{X}
$$




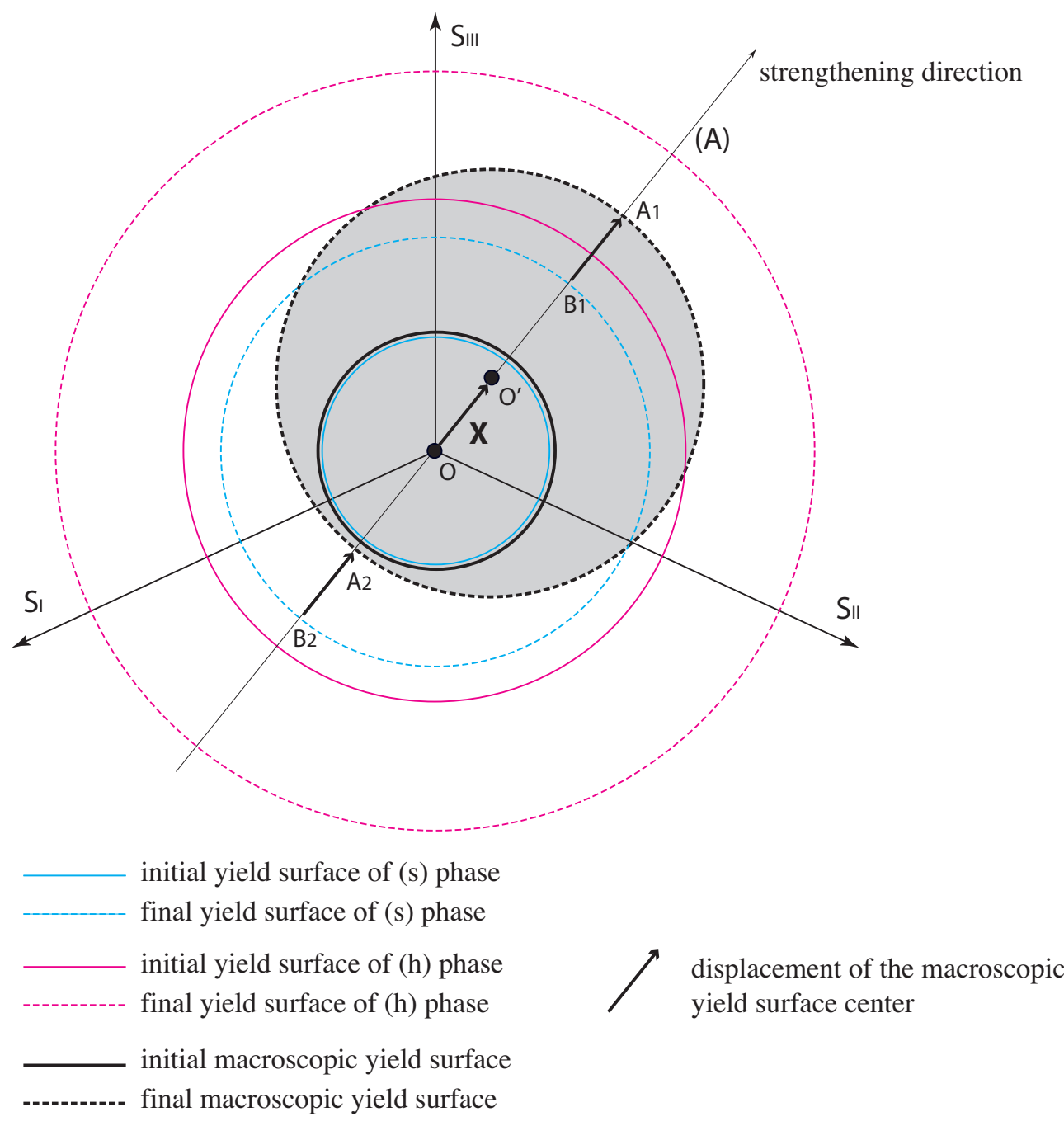

Figure 7: Initial and final (after plastic straining) yield surface of soft and hard phase (in color); Initial and final macroscopic yield surface (in black). Ratio chosen for the drawing is 50\% hard and $50 \%$ soft phases.

The kinematic hardening is finally given by an euclidian difference between macroscopic deviatoric stress and the deviatoric stress within the soft phase ${ }^{2}$ :

$$
\mathbf{X}=\mathbf{S}-\mathbf{S}_{S}
$$

It was previously stated that $\mathbf{D}_{s}$ is the residual stress tensor within the soft phase (12). It is now shown that the kinematic hardening is directly associated to the residual stress within the

\footnotetext{
${ }^{2}$ For micro macro modeling, the same approach leads to define the kinematic hardening as the difference between macroscopic deviatoric stress and the deviatoric stress within the grain exhibiting the lowest yield strength (maximal Schmid factor).
} 
soft phase, so that:

$$
\mathbf{X}=\left(\left(\mathbb{I}-\mathbb{B}_{s}\right): \mathbf{\Sigma}-\frac{1}{3} \operatorname{tr}\left(\left(\mathbb{I}-\mathbb{B}_{s}\right): \mathbf{\Sigma}\right) \mathbf{I}\right)-\left(\mathbf{D}_{s}-\frac{1}{3} \operatorname{tr}\left(\mathbf{D}_{s}\right) \mathbf{I}\right)
$$

or

$$
\mathbf{X}=\frac{f_{h}}{f_{s}}\left(\left(\mathbb{B}_{h}-\mathbb{I}\right): \mathbf{\Sigma}-\frac{1}{3} \operatorname{tr}\left(\left(\mathbb{B}_{h}-\mathbb{I}\right): \mathbf{\Sigma}\right) \mathbf{I}\right)+\frac{f_{h}}{f_{s}}\left(\mathbf{D}_{h}-\frac{1}{3} \operatorname{tr}\left(\mathbf{D}_{h}\right) \mathbf{I}\right)
$$

Assuming that the volume fraction of hard and soft phases, stiffness, Hill, and applied stress tensors are known, an experimental estimation of the quantity $\mathbf{X}$ allows to define the stress field in the two phases except for its hydrostatic part. Considering a homogeneous stiffness medium $\left(\mathbb{C}=\mathbb{C}_{s}=\mathbb{C}_{h}\right.$ ), we get $\mathbb{B}_{s}=\mathbb{B}_{h}=\mathbb{I}$ so that the expression of the kinematic hardening is simplified in:

or

$$
\mathbf{X}=-\left(\mathbf{D}_{s}-\frac{1}{3} \operatorname{tr}\left(\mathbf{D}_{s}\right) \mathbf{I}\right)
$$

$$
\mathbf{X}=\frac{f_{h}}{f_{s}}\left(\mathbf{D}_{h}-\frac{1}{3} \operatorname{tr}\left(\mathbf{D}_{h}\right) \mathbf{I}\right)
$$

A final stronger simplification is obtained if the medium is considered as isotropic because residual stress tensors are deviatoric too, leading to:

$$
\mathbf{X}=-\mathbf{D}_{s}
$$

or

$$
\mathbf{X}=\frac{f_{h}}{f_{s}} \mathbf{D}_{h}
$$

Conversely, we can get $\mathbf{D}_{s}$ and $\mathbf{D}_{h}$ from the knowledge of $\mathbf{X}$ so that we know the local stresses $\sigma_{s}$ and $\sigma_{h}$. In isotropic condition, one gets:

$$
\begin{gathered}
\sigma_{s}=\boldsymbol{\Sigma}-\mathbf{X} \\
\boldsymbol{\sigma}_{h}=\boldsymbol{\Sigma}+\frac{f_{s}}{f_{h}} \mathbf{X}
\end{gathered}
$$

\subsubsection{Application to tensile strengthening of isotropic material}

A tensile loading of an isotropic material with homogeneous stiffness is considered along axis $\vec{x}$ leading to an axial plastic deformation $E^{p}$ (figure 8). Material can be reloaded along the same direction so that the macroscopic stress tensor of magnitude $\Sigma$ is:

$$
\Sigma=\left(\begin{array}{ccc}
\Sigma & 0 & 0 \\
0 & 0 & 0 \\
0 & 0 & 0
\end{array}\right)_{(\vec{x}, \vec{y}, \vec{z})}
$$

The macroscopic plastic strain tensor is constant, diagonal and deviatoric.

$$
\mathbf{E}^{p}=\left(\begin{array}{ccc}
E^{p} & 0 & 0 \\
0 & -\frac{E^{p}}{2} & 0 \\
0 & 0 & -\frac{E^{p}}{2}
\end{array}\right)
$$




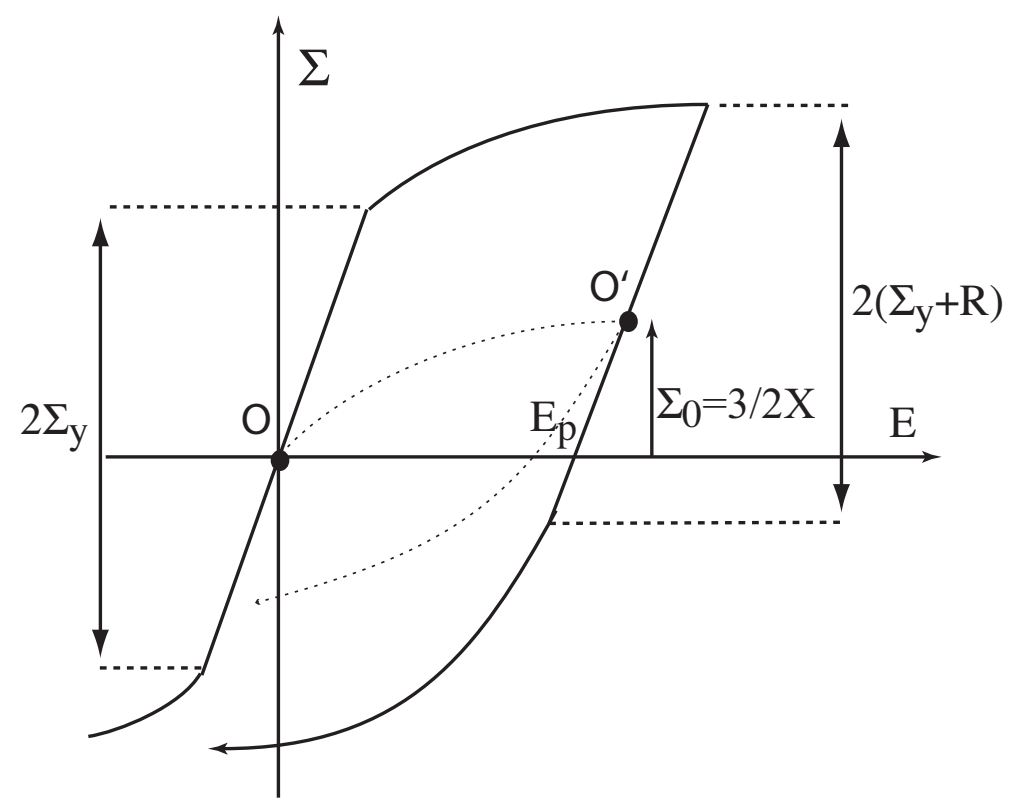

Figure 8: Tensile/compressive stress-strain diagram: associated kinematic and isotropic variables.

Following the Prager or Armstrong-Frederick rules, kinematic hardening is collinear with the macroscopic plastic strain tensor:

$$
\mathbf{X}=\left(\begin{array}{ccc}
X & 0 & 0 \\
0 & -\frac{1}{2} X & 0 \\
0 & 0 & -\frac{1}{2} X
\end{array}\right)
$$

The center of the new yield surface $O^{\prime}$ is given by $\Sigma_{0}=\frac{3}{2} X$, as illustrated in Figure 8 .

\subsection{Magnetic modeling}

We consider a plastically strained material composed of hard $(h)$ and soft $(s)$ phases. We assume that the macroscopic stress $\boldsymbol{\Sigma}$, kinematic hardening $\mathbf{X}$ and volume fraction of $s$ and $h$ phases are known. We assume on the other hand that the macroscopic field $\vec{H}$ is given. The distribution of phases is supposed isotropic so that the shape considered in the localization procedure is spherical. Average behaviors are consequently isotropic but only the behaviors in the strengthening direction are considered in the modeling.

\subsubsection{Magnetic field and stress localization}

The presence of two phases with two different susceptibilities creates a local perturbation of the magnetic field leading to a local demagnetizing field. In this condition, the local fields are not the same as the mean field. In the case of spheroidal inclusion (distribution) [43], the field is demonstrated as homogeneous on each phase and can analytically be calculated. Considering on the other hand a linear susceptibility of average medium $\chi_{m}$, the local magnetic field in the hard and soft phases (denoted $\vec{H}_{h}$ and $\vec{H}_{s}$ respectively) are given by: 


$$
\begin{aligned}
\vec{H}_{s} & =\vec{H}+\frac{1}{3+2 \chi_{m}}\left(\vec{M}-\vec{M}_{s}\right) \\
\vec{H}_{h} & =\vec{H}+\frac{1}{3+2 \chi_{m}}\left(\vec{M}-\vec{M}_{h}\right)
\end{aligned}
$$

where $\vec{M}$ is the average magnetization, $\vec{M}_{s}$ and $\vec{M}_{h}$ are the local magnetization in the soft and hard phases. Extension to nonlinear behavior involves to use the sequent susceptibility for the definition of $\chi_{m}$.

$$
\chi_{m}=\|\vec{M}\| /\|\vec{H}\|
$$

Averaging operations give:

$$
\vec{H}=f_{s} \vec{H}_{s}+f_{h} \vec{H}_{h} \quad \text { and } \quad \vec{M}=f_{s} \vec{M}_{s}+f_{h} \vec{M}_{h}
$$

The stress fields within the two phases have been previously defined by $\sigma_{s}$ and $\sigma_{h}$ in equation (24) for isotropic condition. Magnetostrictive deformation rigorously modifies these stress field due to supplementary strain incompatibilities. If homogeneous isotropic elastic properties (Young's modulus $E$ and Poisson's ratio $v$ ) and additivity of deformation (total deformation = elastic deformation + magnetostrictive deformation) are considered, equation 31 gives the supplementary residual stress field that must be considered as applied on a phase $i$ [46]:

$$
\sigma_{i}^{r}=\frac{E(7-5 v)}{15\left(1-v^{2}\right)}\left(\mathbf{E}^{\mu}-\epsilon_{i}^{\mu}\right)
$$

where $\boldsymbol{\epsilon}_{i}^{\mu}$ and $\mathbf{E}^{\mu}$ denote the local and average magnetostriction strain tensor respectively. Since plastic straining and magnetostriction do not occur at the same time and that magnetostriction leads to a usually low deformation level $\left(<10^{-5}\right)$, a superposition can be applied to define the stress field in the hard and soft phases, leading to:

$$
\begin{gathered}
\sigma_{s}=\boldsymbol{\Sigma}-\mathbf{X}+\frac{E(7-5 v)}{15\left(1-\nu^{2}\right)}\left(\mathbf{E}^{\mu}-\boldsymbol{\epsilon}_{s}^{\mu}\right) \\
\sigma_{h}=\mathbf{\Sigma}+\frac{f_{s}}{f_{h}} \mathbf{X}+\frac{E(7-5 v)}{15\left(1-\nu^{2}\right)}\left(\mathbf{E}^{\mu}-\boldsymbol{\epsilon}_{h}^{\mu}\right)
\end{gathered}
$$

Averaging operations lead to :

$$
\mathbf{E}^{\mu}=f_{s} \epsilon_{s}^{\mu}+f_{h} \epsilon_{h}^{\mu} \quad \text { and } \quad \boldsymbol{\Sigma}=f_{s} \boldsymbol{\sigma}_{s}+f_{h} \boldsymbol{\sigma}_{h}
$$

The magnetostrictive part of residual stress will be considered in the modeling detailed hereafter but its impact on the overall behavior remains low comparing to the effect of residual stresses associated with plastic straining.

\subsubsection{Multidomain modeling}

The modeling uses a two-scale (magnetic domains and grain scale) reversible model introduced in [46] and used to model a biphasic Fe-Al-B material. This section will review its main characteristics.

In this model, the behavior of the whole phase is supposed to be described by the behavior of only one grain of this phase, after an appropriate choice of loading (magnetic and mechanical) direction. Indeed, as explained in [56], the behavior of an isotropic polycrystal is necessarily given by a loading along a specific direction inside the standard crystallographical triangle. This 
direction is not the average direction and may change with stress or magnetic field level because behaviors are usually non-linear. Nevertheless it is possible, as a first approximation, to consider that an isotropic behavior is roughly obtained when the loading is corresponding to the average direction of the standard triangle. In case of cubic symmetry, this direction is defined by spherical angles $(\phi, \theta)=\left(38.81^{\circ}, 77.54^{\circ}\right)$. Following the proposition of [57], a set of 34635 directions equally distributed in the unit sphere has been used to describe the potential domain directions of this grain. At each domain $\alpha$ of direction $\vec{\gamma}_{\alpha}=\gamma_{u} \vec{e}_{u}$ there is a corresponding magnetization vector $\vec{M}_{\alpha}=M_{s} \vec{\gamma}_{\alpha}$, and a magnetostriction tensor $\boldsymbol{\epsilon}_{\alpha}^{\mu}$ defined in equation (34) in the crystal frame $\left(\mathrm{CF}=\left(\vec{e}_{1}, \vec{e}_{2}, \vec{e}_{3}\right)\right)$.

$$
\boldsymbol{\epsilon}_{\alpha}^{\mu}=\frac{3}{2}\left(\begin{array}{ccc}
\lambda_{100}^{i}\left(\gamma_{1}^{2}-\frac{1}{3}\right) & \lambda_{111}^{i} \gamma_{1} \gamma_{2} & \lambda_{111}^{i} \gamma_{1} \gamma_{3} \\
\lambda_{111}^{i} \gamma_{1} \gamma_{2} & \lambda_{100}^{i}\left(\gamma_{2}^{2}-\frac{1}{3}\right) & \lambda_{111}^{i} \gamma_{2} \gamma_{3} \\
\lambda_{111}^{i} \gamma_{1} \gamma_{3} & \lambda_{111}^{i} \gamma_{2} \gamma_{3} & \lambda_{100}^{i}\left(\gamma_{3}^{2}-\frac{1}{3}\right)_{C F}
\end{array}\right)
$$

$\lambda_{100}^{i}$ and $\lambda_{111}^{i}$ are the magnetostriction constants of the phase $i$. The single crystal corresponding to the phase $i$ is submitted to a magnetic field $H_{i}$ and uniaxial stress $\sigma_{i}$ applied in direction $\vec{x}=\cos \phi \sin \theta \vec{e}_{1}+\sin \phi \sin \theta \vec{e}_{2}+\cos \theta \vec{e}_{3}$. The contribution to the free energy of a magnetic domain $W_{\alpha}$ are the magnetostatic energy $W_{\alpha}^{H}$, the magnetocrystalline energy $W_{\alpha}^{K}$ and the magnetoelastic energy $W_{\alpha}^{\sigma}(35)$. A configuration energy (36) using a fictitious configuration stress at the phase scale $\sigma_{i}^{\text {conf }}$ may have to be employed to take account of a possible disequilibrium in the initial domain distribution (see [44] for more details about the configuration terms).

$$
\begin{gathered}
W_{\alpha}^{H}=-\mu_{0} \vec{H}_{i} \cdot \vec{M}_{\alpha} \quad W_{\alpha}^{\sigma}=-\sigma_{i}: \epsilon_{\alpha}^{\mu} \quad W_{\alpha}^{K}=K_{1}\left(\left(\gamma_{1} \gamma_{2}\right)^{2}+\left(\gamma_{2} \gamma_{3}\right)^{2}+\left(\gamma_{1} \gamma_{3}\right)^{2}\right) \\
W_{\alpha}^{\text {conf }}=-\boldsymbol{\sigma}_{i}^{\text {conf }}: \boldsymbol{\epsilon}_{\alpha}^{\mu}
\end{gathered}
$$

The volume fraction $f_{\alpha}$ of a domain $\alpha$ is calculated in function of the free energies using formula (37) derived from the Boltzmann function:

$$
f_{\alpha}=\frac{\exp \left(-A_{s} \cdot W_{\alpha}\right)}{\int_{\alpha} \exp \left(-A_{s} \cdot W_{\alpha}\right) d \alpha}
$$

$A_{s}$ is a parameter related to the initial susceptibility $\chi_{0}^{i}$ of the magnetization curve for the considered phase $i$ :

$$
A_{s}=\frac{3 \chi_{0}^{i}}{\mu_{0} M_{s}^{2}}
$$

By employing $f_{\alpha}$ it is possible to calculate the average magnetization $M_{i}\left(H_{i}, \sigma_{i}\right)$ and the average magnetostriction $\epsilon_{i}^{\mu}\left(H_{i}, \sigma_{i}\right)$ in the direction of applied field/stress by using equations (39).

$$
\vec{M}_{i}=\frac{1}{N} \int_{\alpha} f_{\alpha} \vec{M}_{\alpha} d \alpha \quad \epsilon_{i}^{\mu}=\frac{1}{N} \int_{\alpha} f_{\alpha} \epsilon_{\alpha}^{\mu} d \alpha \quad M_{i}=\vec{M}_{i} \cdot \vec{x} \quad \epsilon_{i}^{\mu}={ }^{t} \vec{x} \cdot \epsilon_{i}^{\mu} \cdot \vec{x} .
$$

Both hard and soft phases are modeled separately. Self consistent localization rules (28) and (32) are used for the calculation of the magnetic field and stress at the phase scale. The 
magnetization and magnetostriction are estimated at each calculation loop and introduced inside the self consistent rules. The calculation is iterated until convergence of average quantities. Results are given in term of average magnetization and magnetostriction following:

$$
\begin{aligned}
\vec{M}(\vec{H}, \boldsymbol{\Sigma}, \mathbf{X}) & =f_{s} \vec{M}_{s}\left(\vec{H}_{s}, \boldsymbol{\sigma}_{s}\right)+f_{h} \vec{M}_{h}\left(\vec{H}_{h}, \boldsymbol{\sigma}_{h}\right) \\
\mathbf{E}^{\mu}(\vec{H}, \boldsymbol{\Sigma}, \mathbf{X}) & =f_{s} \boldsymbol{\epsilon}_{s}^{\mu}\left(\vec{H}_{s}, \boldsymbol{\sigma}_{s}\right)+f_{h} \boldsymbol{\epsilon}_{h}^{\mu}\left(\vec{H}_{h}, \boldsymbol{\sigma}_{h}\right)
\end{aligned}
$$

\subsubsection{Equivalent stress}

As indicated in the paragraph above, the stress introduced in the multidomain modeling must be a uniaxial stress. The kinematic hardening tensor and residual stress associated with the magnetostrictive incompatibilities are nonetheless multiaxial. We have to transform the multiaxial expression into an uniaxial equivalent one. The retained transformation is defined in [45] following:

$$
\sigma^{e q}=\frac{3}{2}{ }^{t} \vec{n} \mathbf{s} \vec{n}
$$

where $\mathbf{S}$ is the deviatoric part of the stress tensor and $\vec{n}$ denotes the direction of applied magnetic field.

An equivalent magneto-mechanical stress replaces the multiaxial stresses $\sigma_{s}$ and $\sigma_{h}$ according to the direction of the magnetic loading $\vec{n}=\vec{x}$. The equivalent stress is given by:

$$
\sigma_{i}^{e q}=\frac{3}{2}{ }^{t} \vec{x} \mathbf{s}_{i} \vec{x}
$$

Index $i$ indicates $s$ or $h$ phase. $\mathbf{s}_{i}$ is the deviatoric tensor associated to $\sigma_{s}$ and $\sigma_{h}$ respectively. Since the hydrostatic part of the tensors is not taken into account and the magnetostriction is isochoric, the deviatoric stress in the soft and hard phases is directly related to the kinematic hardening and to the magnetostriction incompatibilities:

$$
\mathbf{s}_{s}=\mathbf{S}-\mathbf{X}+\frac{E(7-5 v)}{15\left(1-v^{2}\right)}\left(\mathbf{E}^{\mu}-\boldsymbol{\epsilon}_{s}^{\mu}\right) \quad \mathbf{s}_{h}=\mathbf{S}+\frac{f_{s}}{f_{h}} \mathbf{X}+\frac{E(7-5 v)}{15\left(1-v^{2}\right)}\left(\mathbf{E}^{\mu}-\boldsymbol{\epsilon}_{h}^{\mu}\right)
$$

allowing us to define the equivalent stress in the soft and hard phases as:

$$
\sigma_{s}^{e q}=\frac{3}{2}{ }^{t} \vec{x}\left(\mathbf{S}-\mathbf{X}+\frac{E(7-5 v)}{15\left(1-v^{2}\right)}\left(\mathbf{E}^{\mu}-\boldsymbol{\epsilon}_{s}^{\mu}\right)\right) \vec{x} \quad \sigma_{h}^{e q}=\frac{3}{2}{ }^{t} \vec{x}\left(\mathbf{S}+\frac{f_{s}}{f_{h}} \mathbf{X}+\frac{E(7-5 v)}{15\left(1-v^{2}\right)}\left(\mathbf{E}^{\mu}-\boldsymbol{\epsilon}_{h}^{\mu}\right)\right) \vec{x}
$$

On the other hand, in case of uniaxial plastic deformation along $\vec{x}$ axis with axial stress $\Sigma$, axial kinematic hardening $3 / 2 X$ and axial magnetostrictions $E^{\mu}, \epsilon_{s}^{\mu}$ and $\epsilon_{h}^{\mu}$, the magnetomechanical equivalent stresses in $s$ and $h$ phases are:

$$
\sigma_{s}^{e q}=\Sigma-\frac{3}{2} X+\frac{E(7-5 v)}{10\left(1-v^{2}\right)}\left(E^{\mu}-\epsilon_{s}^{\mu}\right) \quad \sigma_{h}^{e q}=\Sigma+\frac{f_{s}}{f_{h}} \frac{3}{2} X+\frac{E(7-5 v)}{10\left(1-v^{2}\right)}\left(E^{\mu}-\epsilon_{h}^{\mu}\right)
$$

The following points can be highlighted: 
- In case of iron-based materials (iron-silicon, steels), the magnetostriction amplitude is small so that the contribution of magnetostriction to the equivalent stress is only a few MPa. It can be neglected compared to the kinematic hardening and applied stress magnitudes. The following expressions of equivalent stress are obtained:

$$
\sigma_{s}^{e q} \approx \Sigma-\frac{3}{2} X \quad \sigma_{h}^{e q} \approx \Sigma+\frac{f_{s}}{f_{h}} \frac{3}{2} X
$$

The magnetostriction contribution is not considered in the following points.

- In the unloaded state after plastic deformation, the $s$ phase is submitted to compression, the $h$ phase to tension since $X$ is positive. This result joins the hypotheses of Cullity [2] in order to interpret the results carried out on a plastically strained iron-silicon alloy (the $h$ phase actually corresponds to the grain boundaries of the material). This is in accordance with the results obtained using the microcrystalline approach.

- In order to reduce the equivalent stress in the $s$ phase to zero, a tensile stress must be superimposed:

$$
\Sigma=\Sigma_{0}=\frac{3}{2} X
$$

The equivalent stress in the $h$ phase is however non zero:

$$
\sigma_{h}^{e q}=\frac{\Sigma_{0}}{f_{h}} \neq 0
$$

This result joins the early experimental observations of Langman [51] and some more recent ones [24] for mild steel and non-oriented 3\% $\mathrm{Si}-\mathrm{Fe}$ alloy respectively. For these cases, the $h$ phase corresponds to either the grain boundaries, the pearlite phase in steels, the other grains not suitably oriented for plastic gliding, or dislocation walls and tangles. It should be noted that Iordache [24] observed a recovery of the behavior of laminated $\mathrm{Fe}-3 \% \mathrm{Si}$ after plastic deformation for a superimposed stress higher than $\Sigma_{0}$ (observed for $\left.\Sigma \approx \frac{3}{2} \Sigma_{0}=\frac{9}{4} X\right)^{3}$. We remark that: i) there is not a real recovery of magnetic behavior (all magnetic quantities are lower than the initial ones); ii) the recovery stress corresponds to an extremum of magnetic quantities. This extremum may be related with the nonmonotonic variation of the magnetic behavior at high tensile stress observed with this family of materials.

- In order to annul the equivalent stress in the $h$ phase, a compressive stress must be superimposed:

$$
\Sigma=-\frac{f_{s}}{f_{h}} \Sigma_{0}=-\frac{3}{2} \frac{f_{s}}{f_{h}} X
$$

The equivalent stress in the $s$ phase is however not zero:

$$
\sigma_{s}^{e q}=-\frac{\Sigma_{0}}{f_{h}} \neq 0
$$

\footnotetext{
${ }^{3}$ The author confused the uniaxial and multiaxial kinematic strengthening. $X$ indicates on the one hand the centre position of yield surface and the value of kinematic strengthening in the kinematic stress tensor on the other hand. These two definitions are incompatible.
} 
- A magnetic measurement performed along a direction $\vec{n}$ different from the mechanical loading direction $\vec{x}$ leads to an expression of the equivalent stresses dependent on the angle $\psi$ between the two vectors, following:

$$
\sigma_{s}^{e q}=\left(\Sigma-\frac{3}{2} X\right)\left(\cos ^{2} \psi-\frac{1}{2} \sin ^{2} \psi\right) \quad \sigma_{h}^{e q}=\left(\Sigma+\frac{f_{s}}{f_{h}} \frac{3}{2} X\right)\left(\cos ^{2} \psi-\frac{1}{2} \sin ^{2} \psi\right)
$$

One notable result is that equivalent stress is always zero for $\psi=\operatorname{atan}(\sqrt{2})$. For $\psi=90^{\circ}$ and $\Sigma=0$, the equivalent stresses are half of the magnitude and of opposite sign than for $\psi=0^{\circ}$, leading to the so-called "magneto-plastic anisotropy" introduced in $[17,27,55]$ and observed earlier by Langman [51]. These relationships do not apply if the plasticity is anisotropic. In this case, the kinematic hardening tensor is defined (in the orthotropic principal frame $\left(\vec{u}_{I}, \vec{u}_{I I}, \vec{u}_{I I I}\right)$ with $\left.\vec{u}_{I}=\vec{x}\right)$ by :

$$
\mathbf{X}=\left(\begin{array}{ccc}
X_{I} & 0 & 0 \\
0 & X_{I I} & 0 \\
0 & 0 & X_{I I I}
\end{array}\right)_{\left(\vec{u}_{I}, \vec{u}_{I I}, \vec{u}_{I I I}\right)}
$$

verifying: $X_{I}+X_{I I}+X_{I I I}=0$. Considering $\vec{n} \in\left(\vec{u}_{I}, \vec{u}_{I I}\right)$ for example, the expression of equivalent stresses becomes :

$$
\begin{gathered}
\sigma_{s}^{e q}=\left(\Sigma-\frac{3}{2} X_{I}\right) \cos ^{2} \psi-\left(\frac{1}{2} \Sigma+\frac{3}{2} X_{I I}\right) \sin ^{2} \psi \\
\sigma_{h}^{e q}=\left(\Sigma+\frac{f_{s}}{f_{h}} \frac{3}{2} X_{I}\right) \cos ^{2} \psi-\left(\frac{1}{2} \Sigma-\frac{f_{s}}{f_{h}} \frac{3}{2} X_{I I}\right) \sin ^{2} \psi
\end{gathered}
$$

Such an approach could help to interpret the results obtained by Küpferling [52] who observed some strong differences of magnetic behavior for a pure iron submitted to two different stress paths at the same deformation level.

Another interesting consequence is that assuming an univocal correlation between magnetic or magnetostrictive behavior and equivalent stress, magnetic measurements performed along different directions (two directions at least) would allow an estimation of the $\mathbf{X}$ tensor in a material.

\section{Application to dual phase steel}

\subsection{Material and experimental protocol}

A dual phase steel has been used in the study (DP780 Steel from ArcelorMittal). Its microstructure consisted of about $30 \%$ vol of hard (mechanically) martensite islands dispersed in a soft (ductile) ferritic matrix (figure 9). This composite structure allows for the creation of strong internal stresses during plasticity whose origin is associated with a strong difference in the yield stresses of both phases [58]. The material has the ideal microstructure for the proposed study because it theoretically allows the creation of large magnitude internal stresses at moderate plastic strain level. From a magnetic consideration, the ferritic matrix can be considered as pure iron. Several authors reported that the steel martensite is ferromagnetic but exhibits a susceptibility much lower than the susceptibility of a pearlite-ferrititic steel at the same carbon content and a high coercive field due to a large amount of various metallurgical products and defects [1, 59]. The mixture of the two phases leads to a soft ferromagnetic material. 


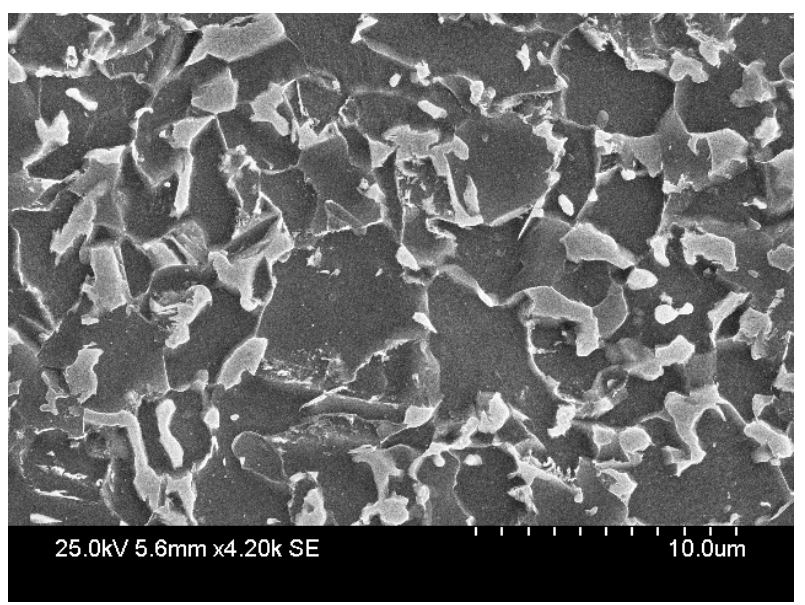

Figure 9: Microstructure of the dual-phase steel - white: martensite islands; black: polycrystalline ferritic matrix.

The samples were $140 \mathrm{~mm}$ long, $12.5 \mathrm{~mm}$ wide, and $3 \mathrm{~mm}$ thick laminations. A MTS uniaxial electrohydraulic machine (displacement controlled) has been used to carry out the uniaxial stress-strain $\Sigma(\mathrm{E})$ behavior of the material. Unloading/reloading tests allowed us to estimate the kinematic $\mathbf{X}$ and isotropic $\mathrm{R}$ hardenings as a function of the plastic strain $E^{p}$ via a Cottrell's method, which is illustrated in Figure 10. The estimation is based on the identification of limits of the yield surface [48]: maximum stress (tensile test) and minimum stress (compression test). Note that this technique requires the yield surface to be exceeded when carrying out the compression test. A strain offset $\delta$ must be chosen on the other hand to detect the non linearity associated with the beginning of plastic flow. The offset chosen during experiments was $\delta=0.5 \times 10^{-4}$.

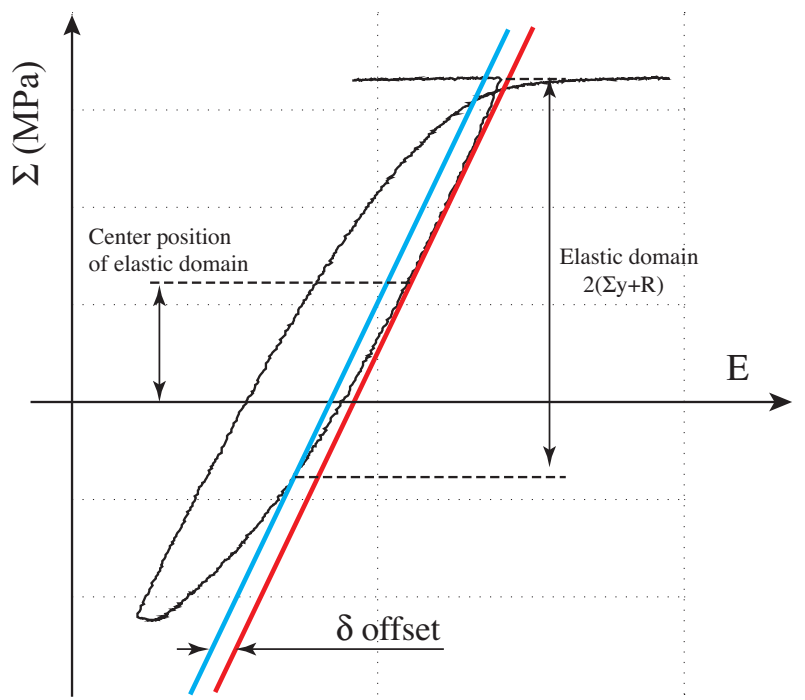

Figure 10: Experimental loading-unloading cycle for the identification of hardening parameters using the Cottrell's method. 
The experimental magnetic device enabled magnetic measurements on plastically strained samples in the unloaded state or under reloaded tensile stress. It was composed of a primary coil to magnetize the sample, an H-coil for the measurement of the magnetic field, a pick-up coil (B-coil) to measure the electromotive force, and a set of longitudinal and transverse strain gauges stuck on both faces of the sheet to estimate the plastic strain level and measure the (longitudinal and transverse) magnetostrictive behavior. Two ferrite yokes were assembled with the sample to close the magnetic circuit and reduce the macroscopic demagnetizing field and the form effect. Measurements have been first performed on unstrained samples providing the reference magnetic and magnetostrictive state. Measurements have been next performed on samples submitted to an increasing plastic deformation level $E^{p}$ : at unloaded state first $(\Sigma=0)$ and under increasing reloaded stress $\Sigma$ below the new yield strength. It has been verified that unloading and re-loading remain in the elastic domain.

Magnetic measurements reported hereafter are the anhysteretic magnetic behavior $M(H)$, the longitudinal and transverse anhysteretic magnetostrictive behavior $\epsilon_{/ /}^{\mu}(M)$ and $\epsilon_{\perp}^{\mu}(M)$, and the hysteretic magnetic behavior $M(H)$ performed at $\mathrm{f}=0.1 \mathrm{~Hz}$. The anhysteretic curves are measured point by point by applying a sinusoidal magnetic field of mean magnitude $H$, and of exponentially decreasing form [60]. Three plastic deformation levels have been investigated: $0.1 \%, 1 \%$ et $3 \%$. Figure 2 and 3 reported in Section 2.1 refer to the reference anhysteretic and cyclic magnetic and magnetostrictive behaviors of the material. Figure 4 refers to the nominal true stress- true strain behavior of the material.

\subsection{Identification of model parameters}

\subsubsection{Identification of kinematic hardening via the Cottrell's method and modeling}

Figure 11 reports the tensile stress-plastic strain behavior of the material. A high level of kinematic hardening $3 / 2 \mathrm{X}$ is observed in accordance with the strong heterogeneity of the material. The isotropic hardening $\mathrm{R}$ is, on the contrary, negligible (a small decrease of true flow stress $\Sigma_{y}+\mathrm{R}$ is observed). Two stages are highlighted in the evolution of kinematic hardening: a first strong increase that saturates immediately reaching $250 \mathrm{MPa}$. The increase of kinematic hardening is then moderated but remains non linear. At high deformation, the evolution is linear.

We choose to model the kinematic hardening by an addition of three terms (54): one Prager linear term $\mathbf{X}_{1}$ (55) and two non-linear Armstrong-Frederick terms $\mathbf{X}_{2}$ (56) and $\mathbf{X}_{3}$ (57) where $\dot{\mathrm{p}}$ indicates the cumulative plastic strain rate (58). Decomposition in two terms (linear and nonlinear) is usual. The second non linear term is employed to simulate the rapid kinematic hardening evolution at the beginning of strengthening. Five parameters have to be identified using the experimental data. Table 1 gives optimized values of modeling parameters (using a least square method). Figure 12 shows a comparison between the modeling results and experimental data. Table 2 regroups the value of $3 / 2 X$ at the plastic strain levels retained for the magnetic measurements. The measurement error for this estimation is about $\pm 20 \mathrm{MPa}$ in accordance with the discrepancies observed with the model.

$$
\mathbf{X}=\mathbf{X}_{1}+\mathbf{X}_{2}+\mathbf{X}_{3}
$$

with:

$$
\begin{gathered}
\dot{\mathbf{X}}_{1}=\frac{2}{3} C_{1} \dot{\mathbf{E}}^{p} \\
\dot{\mathbf{X}}_{2}=\frac{2}{3} C_{2} \dot{\mathbf{E}}^{p}-\frac{1}{\gamma_{2}} \mathbf{X}_{2} \dot{\mathrm{p}}
\end{gathered}
$$




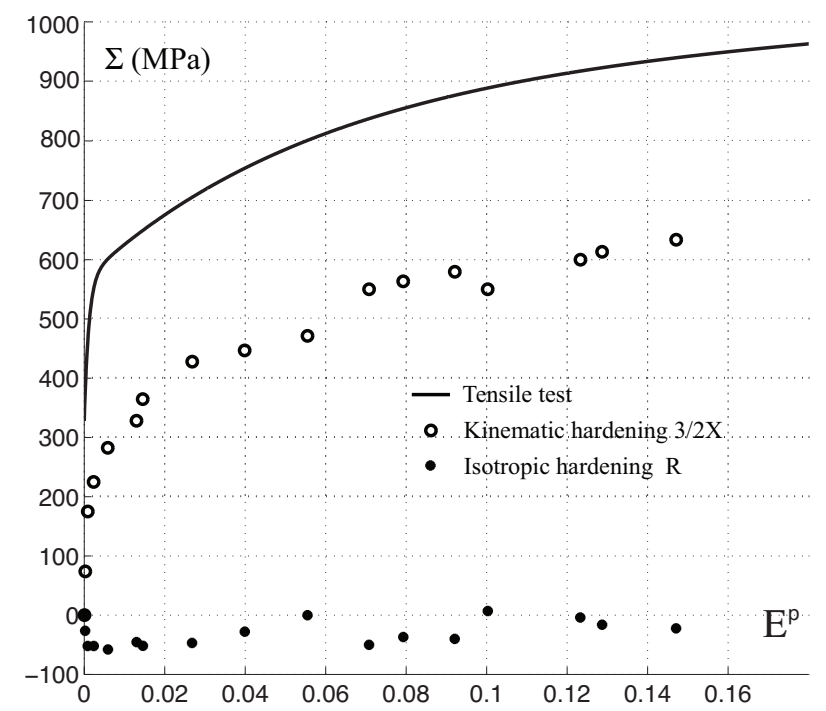

Figure 11: Stress - plastic strain behavior of dual-phase steel - Associated isotropic and kinematic hardening.

\begin{tabular}{|c|c|c|c|c|}
\hline$C_{1}(M P a)$ & $C_{2}(M P a)$ & $\gamma_{2}$ & $C_{3}(M P a)$ & $\gamma_{3}$ \\
\hline 333 & 226667 & 0.00114 & 6000 & 0.059 \\
\hline
\end{tabular}

Table 1: Parameters of the kinematic hardening model of dual-phase steel.

$$
\dot{\mathbf{X}}_{3}=\frac{2}{3} C_{3} \dot{\mathbf{E}}^{p}-\frac{1}{\gamma_{3}} \mathbf{X}_{3} \dot{p}
$$

with

$$
\dot{\mathrm{p}}=\sqrt{\frac{2}{3} \dot{\mathbf{E}}^{p}: \dot{\mathbf{E}}^{p}}
$$

\begin{tabular}{|c|c|}
\hline $\mathrm{E}^{p}$ & $3 / 2 \mathrm{X}(\mathrm{MPa})$ \\
\hline \hline 0.001 & $100 \pm 20$ \\
\hline 0.01 & $270 \pm 20$ \\
\hline 0.03 & $400 \pm 20$ \\
\hline
\end{tabular}

Table 2: Estimation of the stress level corresponding to the centre of yield surface $(3 / 2 X)$ at the plastic strain levels retained for the magnetic experiments.

\subsubsection{Identification of the magnetic parameters for each phase}

As indicated in Section 3.3.2 the multidomain model requires parameters for each phase: ferrite and martensite. The parameter identification needs accurate measurements of the magnetic and magnetostrictive behaviors of both materials taken seperately. The behavior of ferrite is assumed to be very close to the behavior of pure iron. The behavior and physical parameters of 


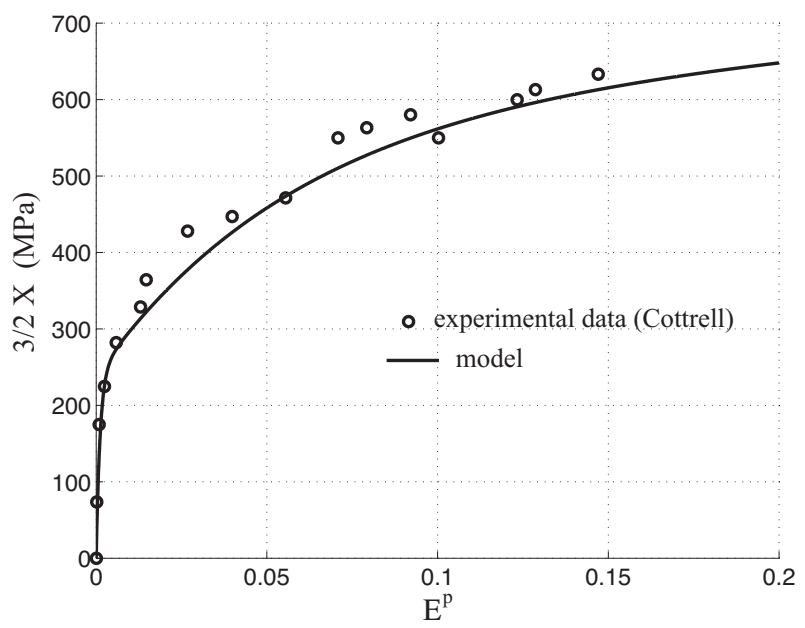

Figure 12: Measurement and modeling of the kinematic hardening

such a material are well documented. The behavior of the martensitic phase is more difficult to obtain and much less well documented in the literature. Indeed martensite seems to be strongly sensitive to the carbon content, alloying elements and heat treatments [59]. A procedure detailed in [9] allowed us to obtain a fully martensitic steel after an appropriate heat treatment of a $0.4 \mathrm{wt} \%$ carbon content mild steel (C38 steel). Microhardness and microstructure of DP steel and C38 martensite have been found to be in good agreement. This material is supposed to exhibit the same magneto-mechanical behavior as the martensite phase in the DP steel.

Magneto-mechanical experiments have been performed on a pure iron sample and on a quenched C38 sample following the experimental procedure detailed in Section 4.1. Figures 13 and 14 show the experimental results obtained for magnetic and longitudinal magnetostrictive behavior without an applied stress. As expected, the behavior of DP steel that has been added in the figure is located between the two other behaviors.

The tables below list the parameters used for the modeling of each phase. Physical parameters for pure iron are well known (magnetocrystalline and magnetostrictive constants, saturation magnetization). $\chi_{0}$ and $\sigma^{\text {conf }}$ are the only parameters optimized to fit properly the magnetization curve for $\chi_{0}$ and the magnetostriction curve for $\sigma^{\text {conf }}$. Physical parameters of martensite are unable to be found in the literature. An isotropic behavior has been supposed for this second phase that reduces the magnetostrictive parameters to one. Its value is optimized to fit properly the magnetostriction curve. $\chi_{0}, M_{s}$ and $K_{1}$ are optimized thanks to the magnetization curve. The magnetocrystalline constant $K_{1}$ has been fixed to a high value to reproduce the high coercivity of the martensite. $M_{s}$ has then been reduced from the theoretical value of pure iron to properly fit the saturation in the measurement range. The configuration energy has not been considered for this material since the optimization of the magnetostriction saturation value is enough to get an accurate restitution of the behavior. Other fixed parameters are the loading angles of the multidomain modeling $(\phi, \theta)=\left(38.81^{\circ}, 77.54^{\circ}\right)$ and Young modulus and Poisson ratio $(E, v)=(200 \mathrm{GPa}$, $0.3)$.

Once the best parameters describing the ferrite and martensite behavior are found, a dual phase medium can be modeled using volume fractions $f_{s}=0.7$ and $f_{h}=0.3$. A fixed point 


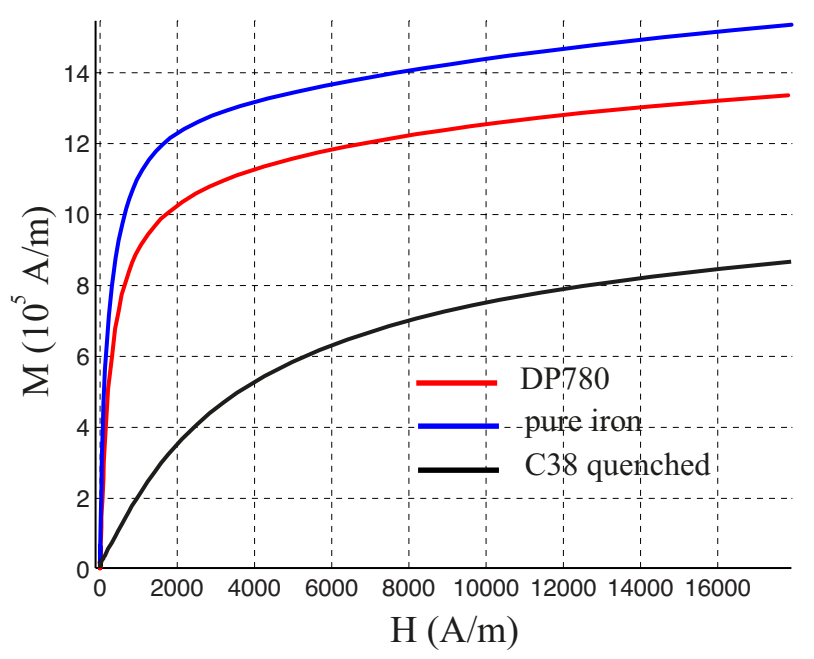

Figure 13: Anhysteretic magnetic behavior of DP780 steel, pure iron and C38 quenched steel.

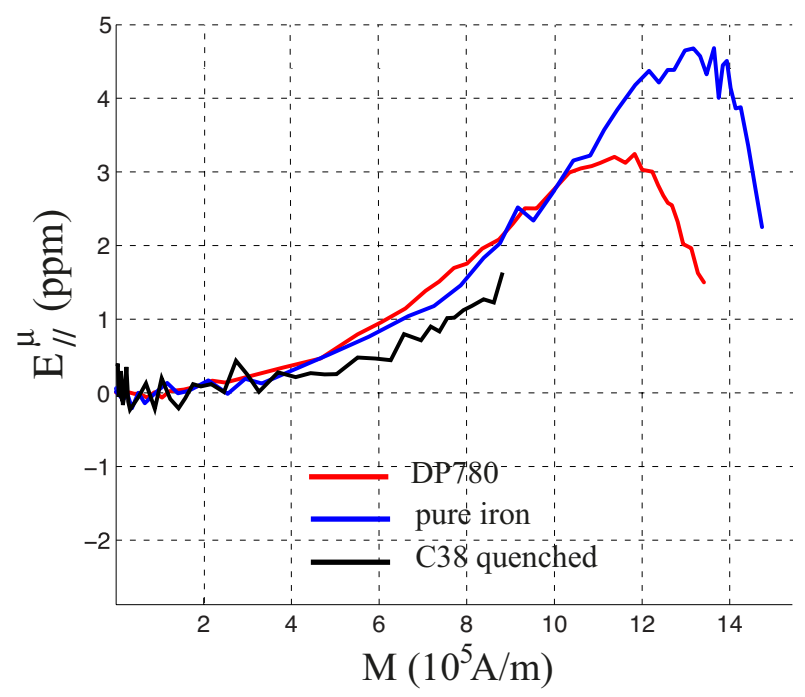

Figure 14: Anhysteretic magnetostrictive behavior of DP780 steel, pure iron and C38 quenched steel.

method is employed to get the average behavior taking account of the stress and magnetic field localization (convergence is reached after less than 10 loops - computation time is about 2.7 seconds per point using Matlab ${ }^{\circledR}$ software implemented in a personal computer). Figures 15 and 16 show the modeling results for magnetization longitudinal magnetostriction curves for the three materials. The modeled behavior for DP780 steel is in very good agreement with the experimental behavior. The bigger discrepancies concern the magnetostriction behavior especially for pure iron at intermediate magnetic fields. 


\begin{tabular}{cccccc} 
Param. & $M_{s}$ & $K_{1}$ & $\lambda_{100} ; \lambda_{111}$ & $\chi_{0}$ & $\sigma^{\text {conf }}$ \\
\hline \hline Value & $1.71 \times 10^{6}$ & 48 & $21 ;-21$ & 2000 & 9 \\
Unit & $\mathrm{A} / \mathrm{m}$ & $\mathrm{kJ} . \mathrm{m}^{-3}$ & $\mathrm{ppm}$ & - & $\mathrm{MPa}$
\end{tabular}

Table 3: Parameters of multidomain modeling for iron $(s)$ phase.

\begin{tabular}{cccccc} 
Param. & $M_{s}$ & $K_{1}$ & $\lambda_{100} ; \lambda_{111}$ & $\chi_{0}$ & $\sigma^{\text {conf }}$ \\
\hline \hline Value & $1.25 \times 10^{6}$ & 500 & $4.8 ; 4.8$ & 150 & 0 \\
Unit & $\mathrm{A} / \mathrm{m}$ & $\mathrm{kJ} . \mathrm{m}^{-3}$ & $\mathrm{ppm}$ & - & $\mathrm{MPa}$
\end{tabular}

Table 4: Parameter for martensite $(h)$ phase.

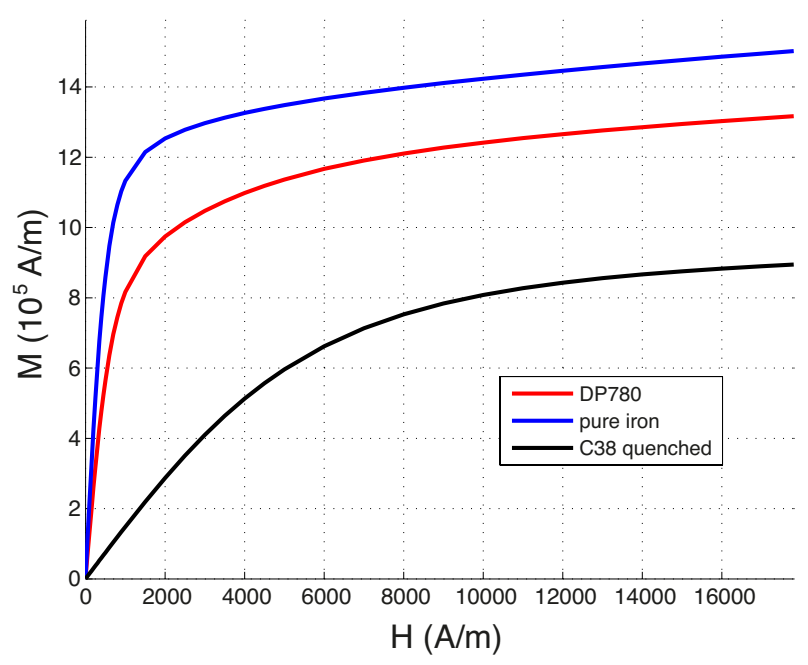

Figure 15: Modeling results - anhysteretic magnetic behavior of DP780 steel, pure iron and C38 quenched steel.

\subsection{Experimental results and modeling}

\subsubsection{Effect of applied stress - elastic domain}

A positive uniaxial stress is applied on the DP steel sample keeping stress below the yield limit. This first test allows to observe the behavior of the material and of the model in a condition where the material is kept in a reversible condition (i.e. $X=0$ ). Experimental and modeling results are plotted successively for a better appreciation of discrepancies. Figure 17a shows the initial state and the effect of stress on the anhysteretic magnetic behavior of the DP steel. The tensile stress has only a moderate influence on the magnetic behavior. This results in a slight increase in the susceptibility before the Villari reversal (beyond $2000 \mathrm{~A} / \mathrm{m}$ ) and a decrease of magnetization after this point, as commonly observed for steels. It can be however noticed that the effect of stress on magnetic susceptibility is non monotonic at high stress intensity. The magnetic behavior at $\Sigma=200 \mathrm{MPa}$ is for example always below the magnetic behavior at $\Sigma=150 \mathrm{MPa}$. 


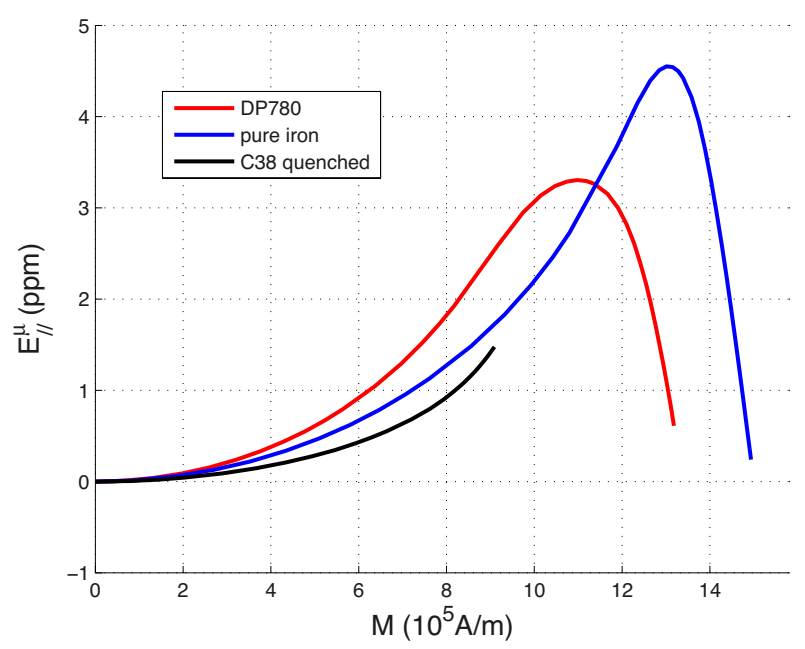

Figure 16: Modeling results - anhysteretic magnetostrictive behavior of DP780 steel, pure iron and C38 quenched steel.

This counterintuitive phenomenon was already observed in iron-silicon [62] or iron-cobalt alloys [63], in contradiction with the classical magneto-elastic effect. It was interpreted and modeled in a multiscale framework in [62] as an effect of stress on the initial domain configuration (demagnetizing stress effect). Except concerning the non-monotony at high tensile stress (the demagnetizing stress effect is not considered in the present paper), the modeling plotted in Figure $17 \mathrm{~b}$ and the experiments are in good agreement.

Figure 18a shows the initial state and the effect of stress on the anhysteretic magnetostriction (longitudinal and transverse) of the DP steel. This behavior is clearly much more sensitive to applied stress than the magnetic behavior. The modeling plotted in Figure 18b gives results in very good agreement with experiments. The saturation level of magnetostriction is however higher for the modeling (from $4.5 \mathrm{ppm}$ for experiments to $5.8 \mathrm{ppm}$ for modeling at $200 \mathrm{MPa}$ ). This difference has to be related to the shift procedure applied to the experimental data as explained in [61]. The plot of experimental and modeled $\Delta \mathrm{E}$ effect in Figure 19 extracted from magnetostriction results allows an illustration of this point. These results show finally the ability of the model to reproduce the effect of stress on the magnetic and magnetostrictive behaviors of the DP steel. It is a prerequisite for modeling the influence of plasticity via a magnetoelastic approach. 


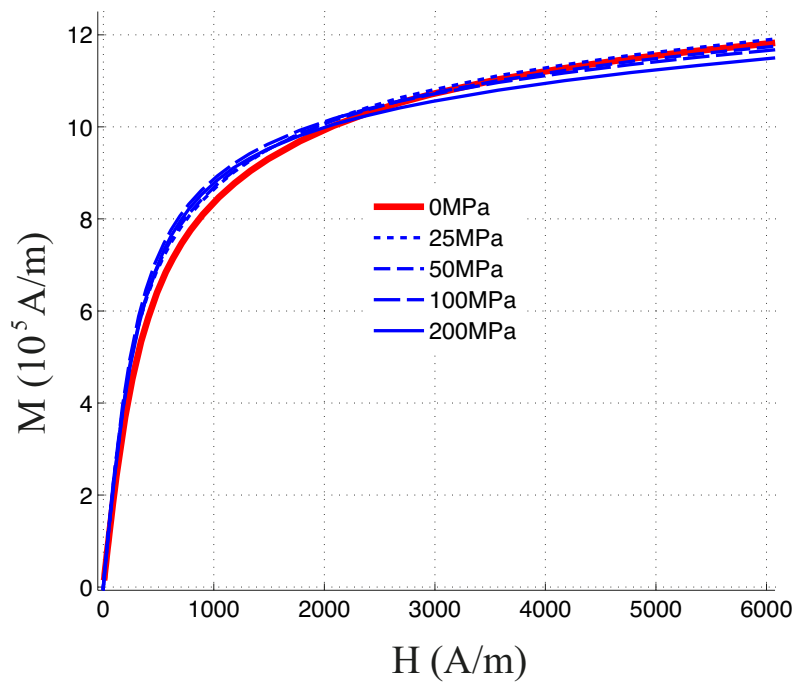

(a) Experiments

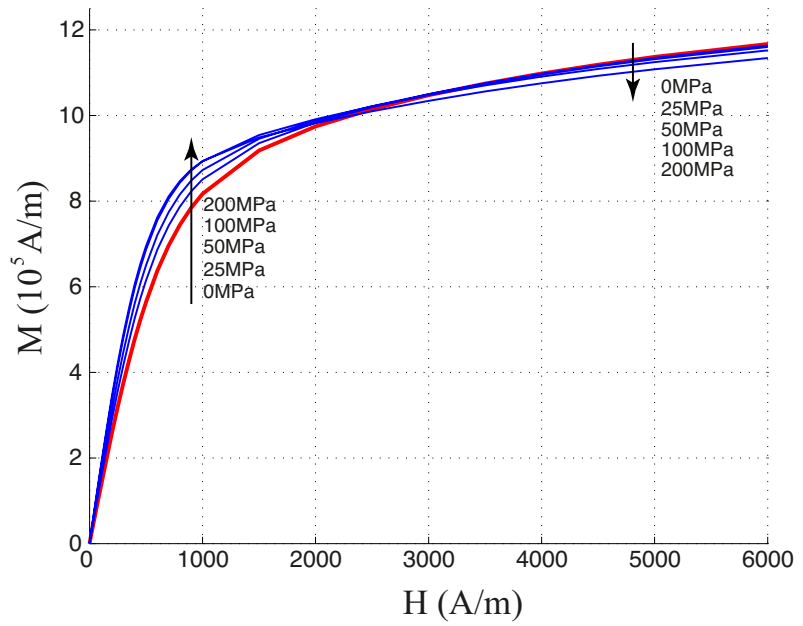

(b) Modeling

Figure 17: Influence of tensile applied stress below yield strength on the anhysteretic magnetic behavior of DP780 steel. 


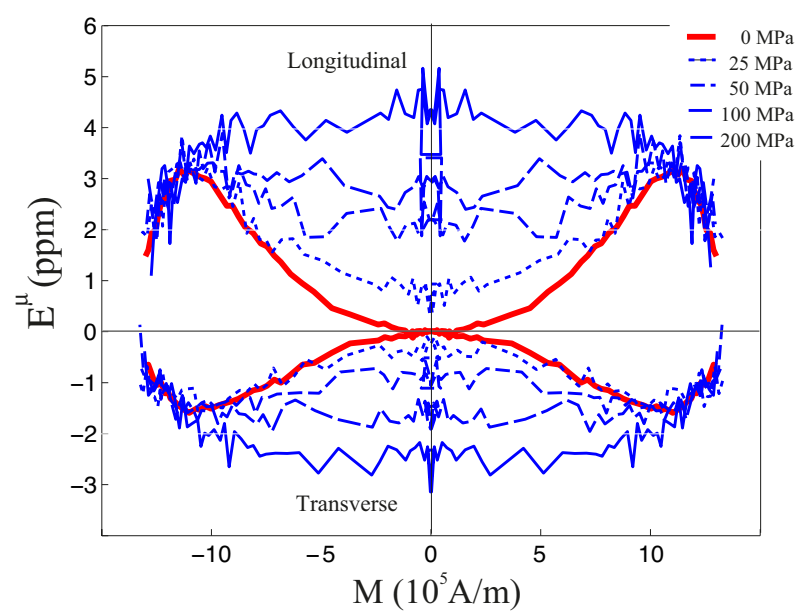

(a) Experiments

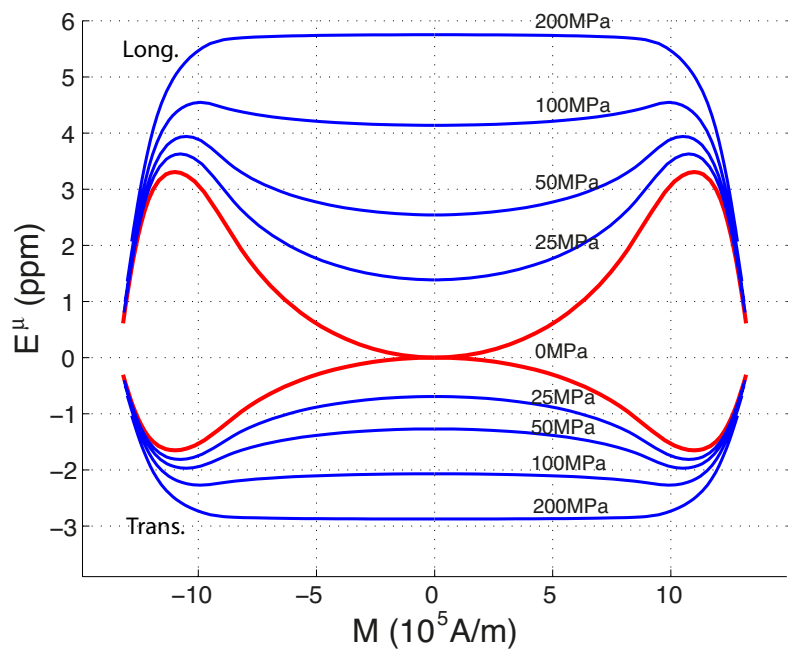

(b) Modeling

Figure 18: Influence of tensile applied stress below yield strength on the anhysteretic magnetostrictive behavior of DP780 steel - Longitudinal: deformation measured in the direction of applied field; Transverse: deformation measured perpendicularly to the direction of applied field. 


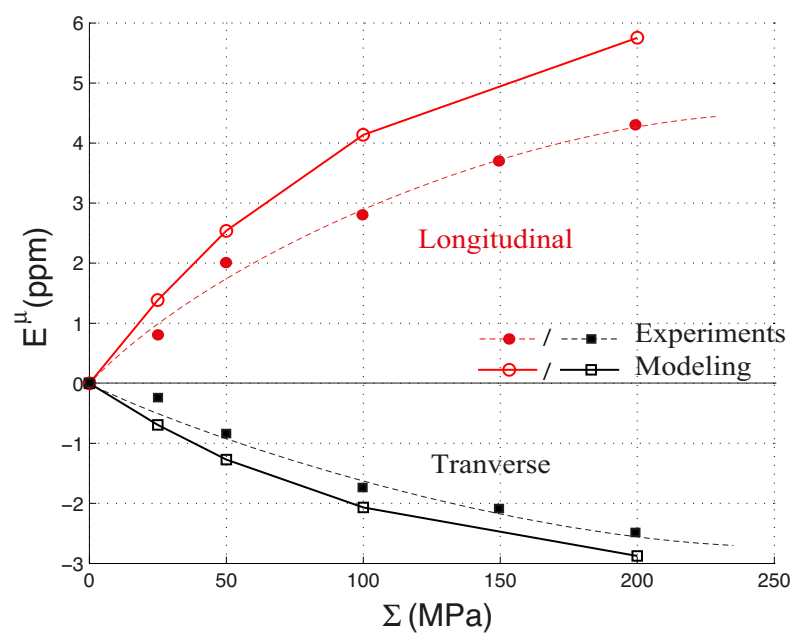

Figure 19: Comparison model/experiments of anhysteteric longitudinal and transverse $\Delta \mathrm{E}$ effect for DP780 steel (extracted from results plotted in Figures 18a and 18b).

\subsubsection{Effect of plastic straining on the magnetic and magnetostrictive behaviors}

Three different specimens of DP780 steel has been deformed up to $0.1 \%, 1 \%$ and $3 \%$ (i.e. $\mathrm{E}_{p}=0.001, \mathrm{E}_{p}=0.01$ and $\mathrm{E}_{p}=0.03$ ). Their magnetic and magnetostrictive behaviors have been measured after plastic deformation at unloaded state first, under applied tensile test next. The uniaxial stress introduced in the modeling of each phase ( $s$ : ferrite; $h$ : martensite) is defined by:

$$
\sigma_{s}^{e q}=\Sigma-\frac{3}{2} X+\frac{E(7-5 v)}{10\left(1-v^{2}\right)}\left(E^{\mu}-\epsilon_{s}^{\mu}\right) \quad \sigma_{h}^{e q}=\Sigma+\frac{f_{s}}{f_{h}} \frac{3}{2} X+\frac{E(7-5 v)}{10\left(1-v^{2}\right)}\left(E^{\mu}-\epsilon_{h}^{\mu}\right)
$$

where $3 / 2 X$ depends on the plastic strain level. Values are reported in the previous Table 2 and come from the Cottrell analysis. The following assumptions are consequently made:

- isotropic distribution of the two phases in the material;

- isotropic distribution of crystallographic orientations in each phase;

- behavior of each phase supposed unchanged with plastic strain.

The last assumption is maybe the weakest because we know that plasticity of the soft phase (ferrite in the present case) is associated with a significant change of dislocation density that may change the behavior of the phase. This contribution is not considered in the modeling.

Figure 20a shows the effect of the three plastic strain levels on the magnetic behavior at unloaded state $(\Sigma=0)$. A strong non linear degradation is observed as reported by many authors in previous works. The corresponding modeling is plotted in Figure 20b giving results in good agreement with experimental ones. Figure 21a and 22a report the associated magnetostrictive behavior (along longitudinal and transverse directions respectively). The plastic strain acts clearly as a compressive stress effect as foreseen by the theoretical approach. Modelings reported in Figure $21 \mathrm{~b}$ and $22 \mathrm{~b}$ give results in good qualitative agreement with experiments. The shape of 
the magnetostriction curves at $\mathrm{E}^{p}=0.03$ nevertheless exhibits some inflexions that do not seem physical. This defect is due to the strong simplifications of the multidomain modeling leading to an dissymmetric rotation of the magnetic moments under stress. This defect disappears when the full multiscale modeling is employed to model the behavior of each phase. The computation time is however thousand of times longer for a relatively small improvement.

As a conclusion of this part, it is possible to confirm that the modeling of the effect of plastic strain on the magnetic behavior through a purely mechanical description (introduction of residual stress field) is possible and gives some very accurate results for plastic straining up to $3 \%$.

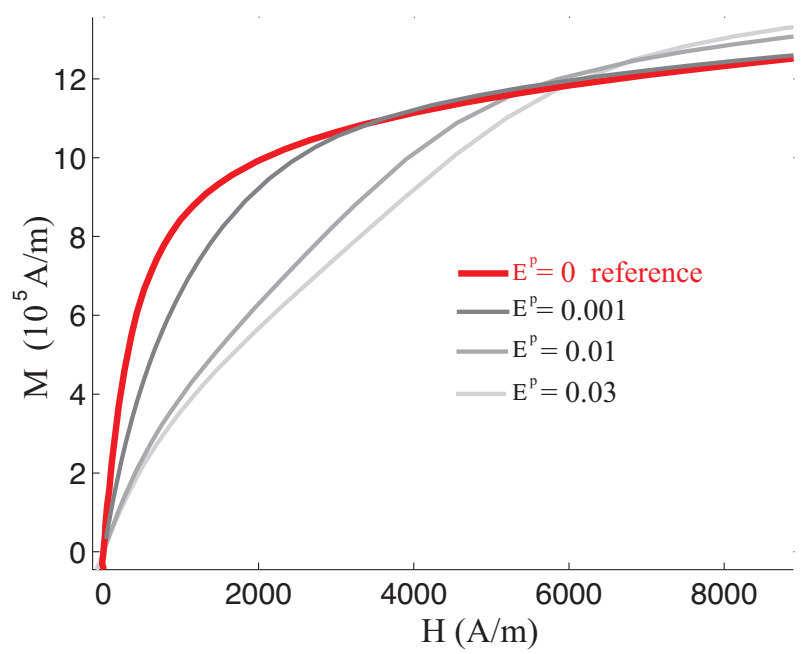

(a) Experiments

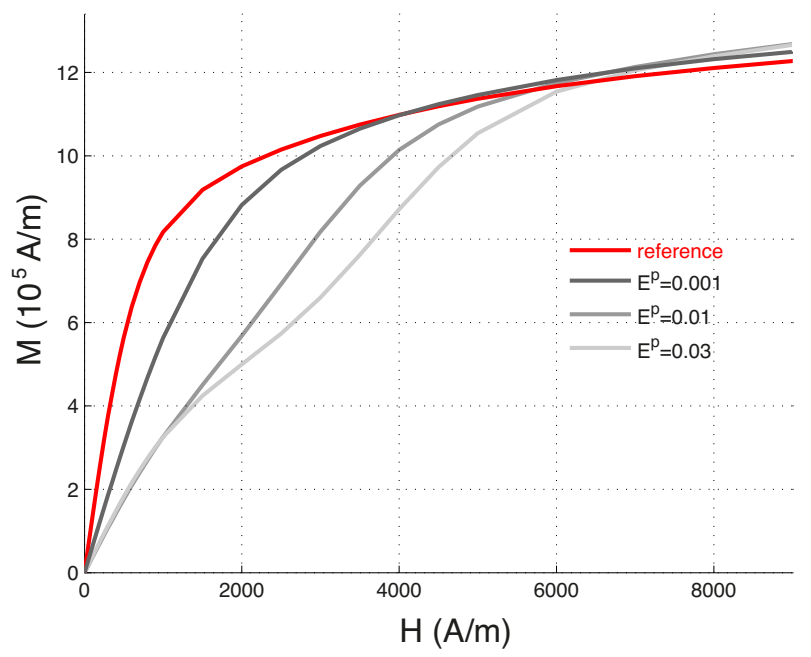

(b) Modeling

Figure 20: Anhysteretic magnetic behavior of plastic strained samples at unloaded state. 


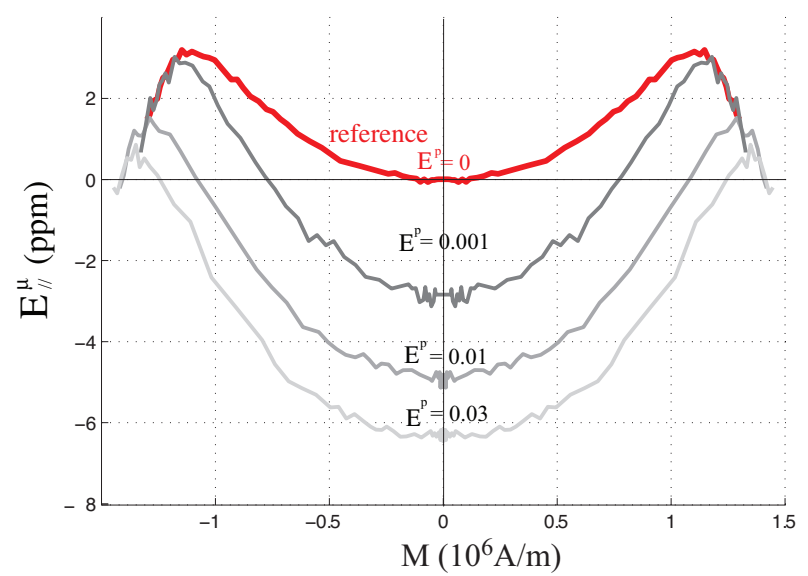

(a) Experiments

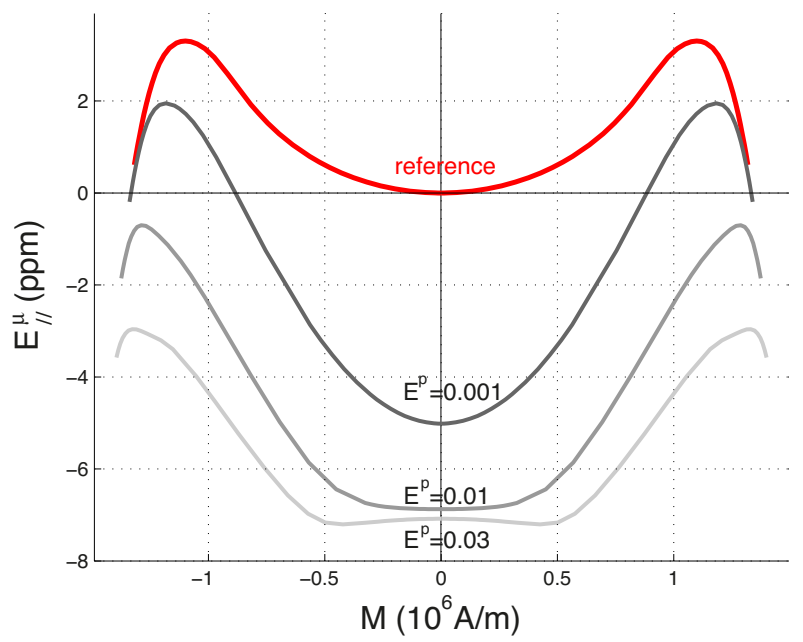

(b) Modeling

Figure 21: Longitudinal anhysteretic magnetostrictive behavior of plastic strained samples at unloaded state. 


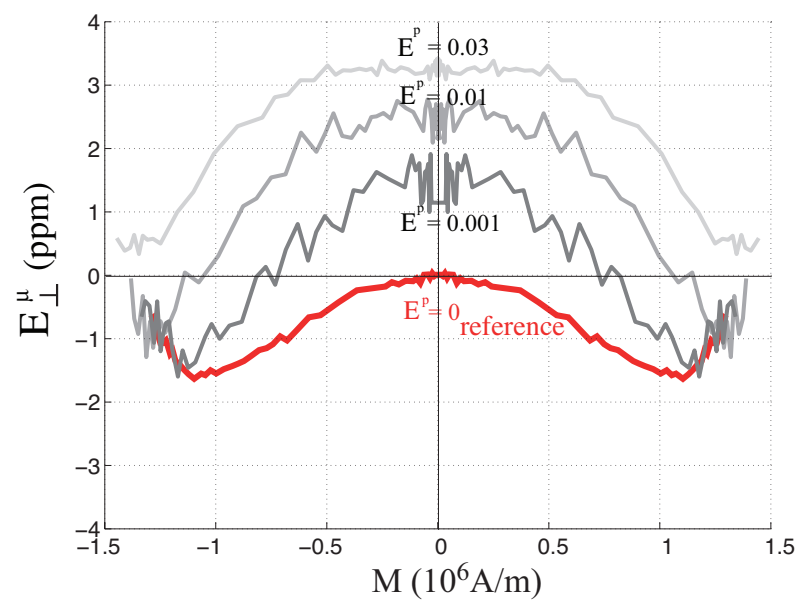

(a) Experiments

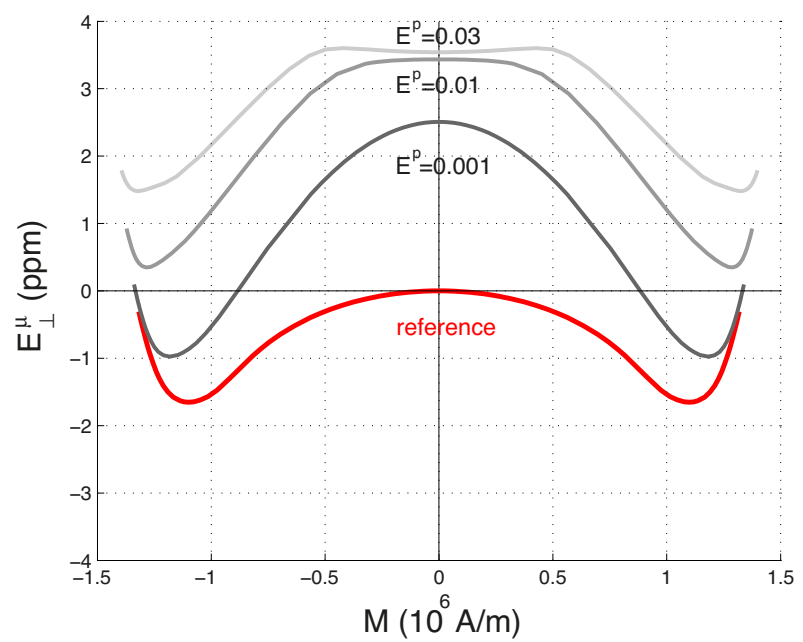

(b) Modeling

Figure 22: Transverse anhysteretic magnetostrictive behavior of plastic strained samples at unloaded state. 


\subsubsection{Effect of plastic straining: reloaded state}

The plastically strained samples are submitted to an increasing level of tensile stress (remaining in the "new" elastic domain). The modeling is obtained by using an increasing value of $\Sigma$ in equation (59). Magnetization, longitudinal and transverse magnetostrictive measurements are performed. Figures 23a, 24a and 25a show the evolution of the magnetic behavior of the sample plastic strained at $0.1 \%, 1 \%$ and $3 \%$ respectively and reloaded at various stress levels indicated in the figures. Figures $23 \mathrm{~b}, 24 \mathrm{~b}$ and $25 \mathrm{~b}$ plot the associated modelings. It is remarkable to observe that the reloading allows to progressively recover the reference $\left(\mathrm{E}^{p}=0\right.$ and $\left.\Sigma=0\right)$ behavior whatever the plastic strain level. This behavior is in agreement with the residual stress origin of the initial degradation associated with plastic strain. Indeed, as explained before, the residual stress in the $s$ phase $\left(\sigma_{e q}^{s} \approx \Sigma-3 / 2 X\right)$ that mostly contributes to the average magnetic behavior, becomes positive for $\Sigma>3 / 2 X$. If the influence of the second phase (lower quantity and lower sensitivity to stress) is negligible, the reference behavior could be reached for an applied stress value theoretically close to $3 / 2 X$ value. Experimental results are roughly in accordance with this interpretation. This conclusion seems less clear for the $0.1 \%$ strained sample where the tested re-loaded stress levels are insufficient. Since the modeling is built using the same principles, it allows a good restitution of the effect of a superimposed applied stress. Particularly, the reference behavior is clearly reached for a stress value close to $3 / 2 X$ that confirms the moderated role of the second phase. This stress denoted $\Sigma_{c}^{\chi}$ is called "magnetic recovery stress". We observe nevertheless that the modeled magnetic behaviors clearly overstep the reference behavior at high stress. This is not observed in the experiments; it should probably be related to the non-monotonic effect of tensile stress already observed but not considered in the modeling. 


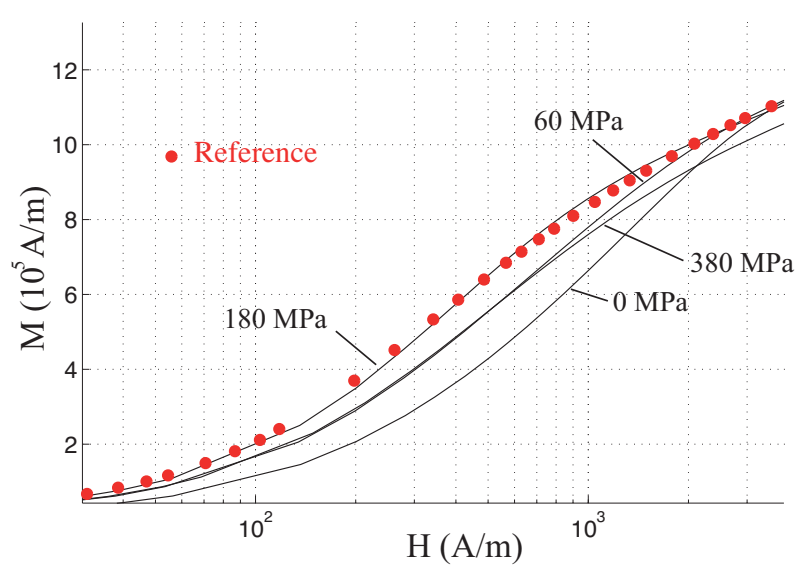

(a) Experiments

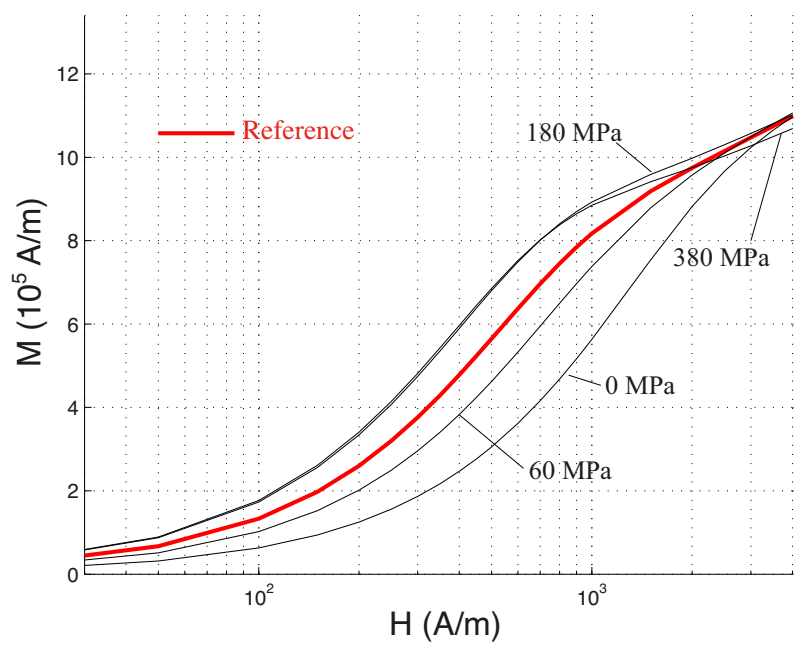

(b) Modeling

Figure 23: Anhysteretic magnetic behavior of the sample plastic strained at $0.1 \%$ and reloaded at various stress levels. 


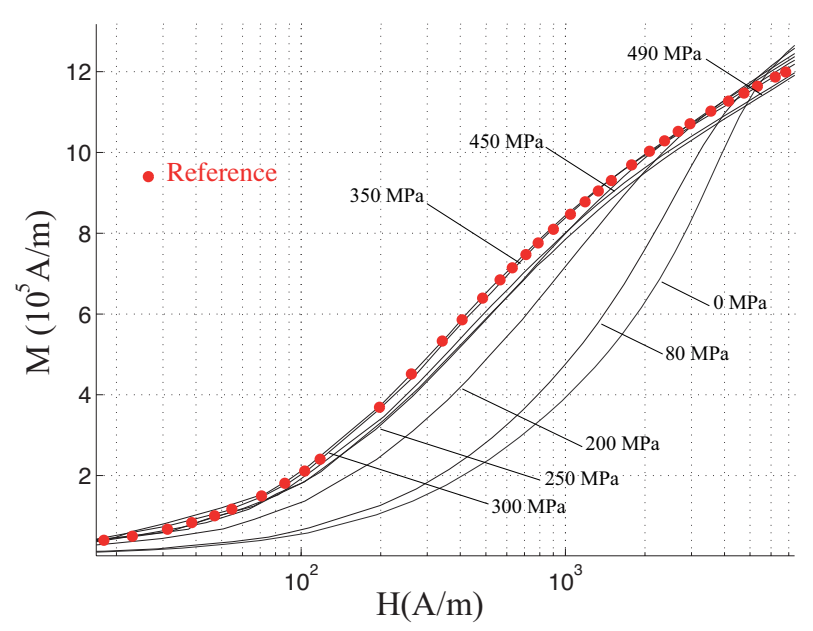

(a) Experiments

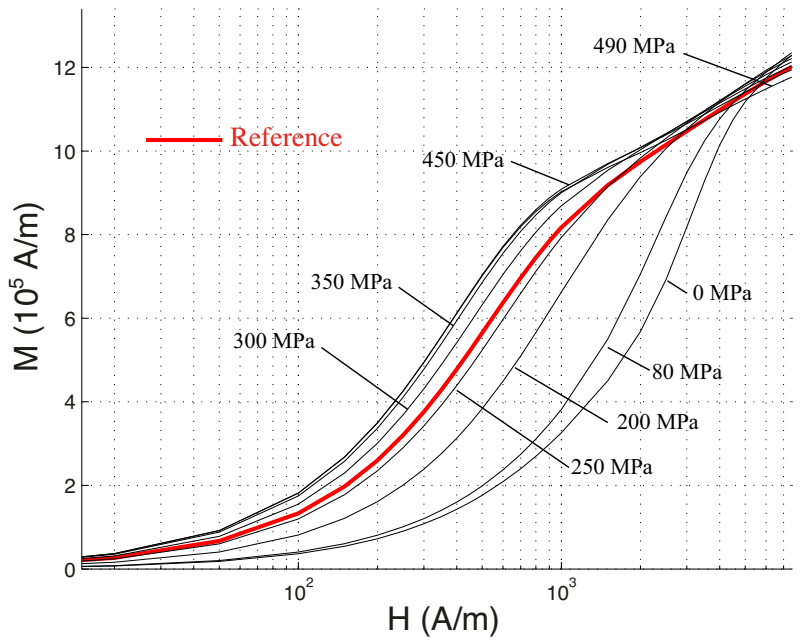

(b) Modeling

Figure 24: Anhysteretic magnetic behavior of the sample plastic strained at $1 \%$ and reloaded at various stress levels. 


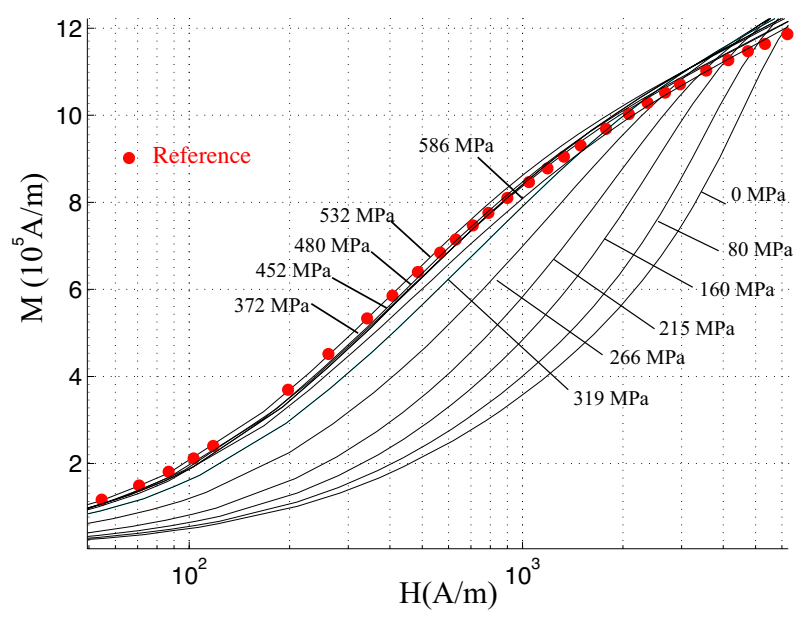

(a) Experiments

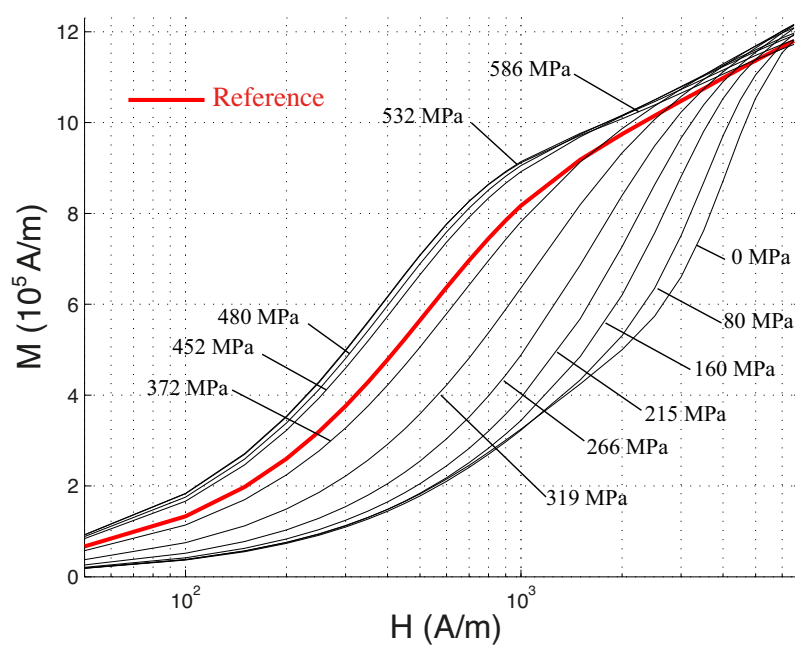

(b) Modeling

Figure 25: Anhysteretic magnetic behavior of the sample plastic strained at 3\% and reloaded at various stress levels.

Figure 26 plots the initial susceptibility $\chi_{0}=(d M / d H)_{H=0}$ of the non-deformed and plastically strained samples as a function of the applied stress for the experiments and modeling. Even if the magnetic behavior cannot be summarized by $\chi_{0}$, this plot allows an easy observation of the effects detailed above. The magnetic recovery stress $\Sigma_{c}^{\chi}$ is clearly identifiable, as the nonmonotonic effect is not reproduced by the modeling. It can be noticed that the global level of initial susceptibility predicted by the model is lower than the experimental susceptibility. This probably comes from the underestimation of the initial susceptibility of the ferrite phase.

Figures 27-32a show the evolution of the longitudinal and transverse magnetostrictive behaviors of the sample plastic strained at $0.1 \%, 1 \%$ and $3 \%$ respectively and reloaded at various stress levels indicated in the figures. Figures $27-32 \mathrm{~b}$ plot the associated modelings in good global 


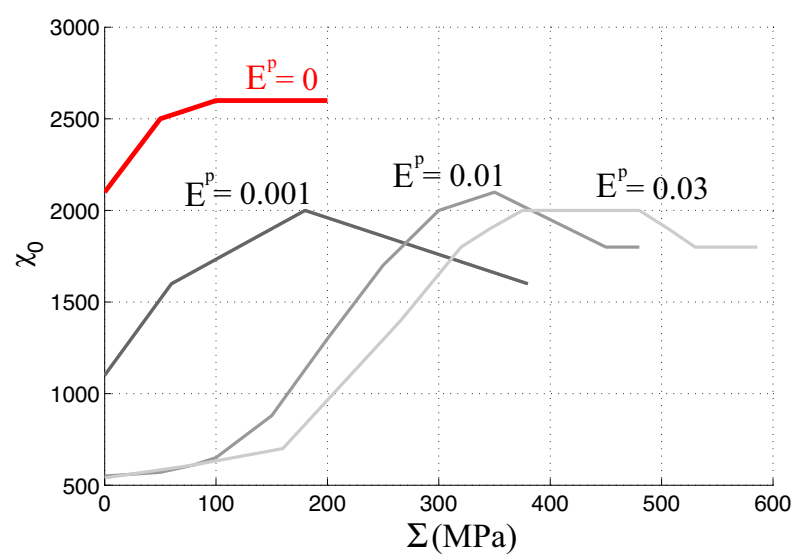

(a) Experiments

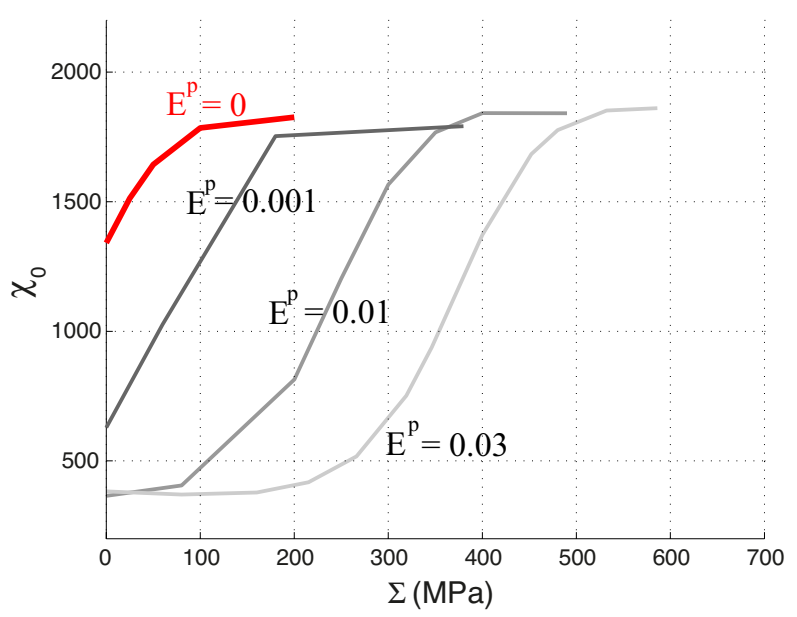

(b) Modeling

Figure 26: Initial susceptibility of the samples as function of the reloaded stress.

agreement with the experiments. As for the magnetic behavior, a critical stress allows to recover the initial magnetostrictive behavior of the material. This "magnetostriction recovery stress" is denoted $\Sigma_{c}^{\chi}$ and values are close to $3 / 2 X$ as observed for the magnetic behavior. Below $\Sigma_{c}^{\mu}$, the material behaves like an unstrained material submitted to a compression. Above $\Sigma_{c}^{\mu}$, the material behaves like an unstrained material submitted to tensile stress. The phenomenon reaches a saturation stage at higher levels of applied stress. 


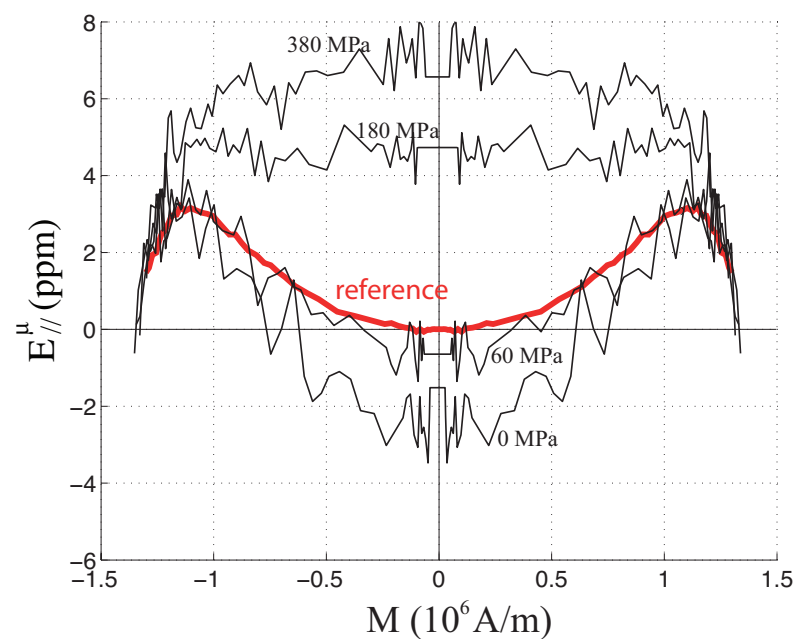

(a) Experiments

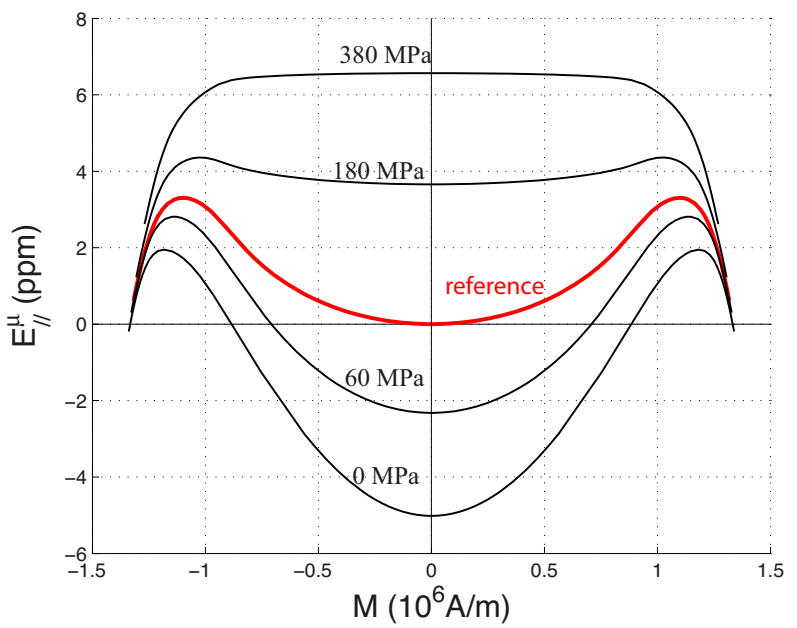

(b) Modeling

Figure 27: Anhysteretic longitudinal magnetostrictive behavior of the sample prestrained at $0.1 \%$ and reloaded at various stress levels. 


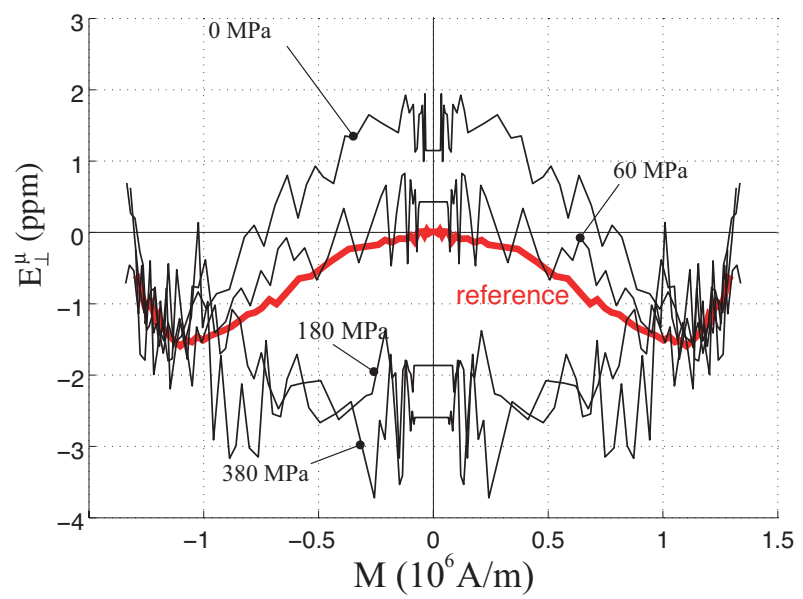

(a) Experiments

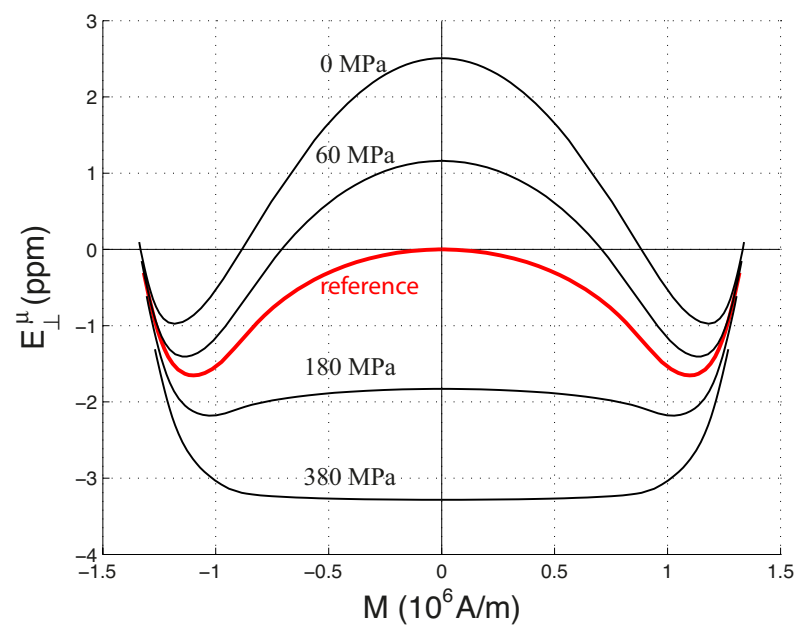

(b) Modeling

Figure 28: Anhysteretic transverse magnetostrictive behavior of the sample prestrained at $0.1 \%$ and reloaded at various stress levels. 


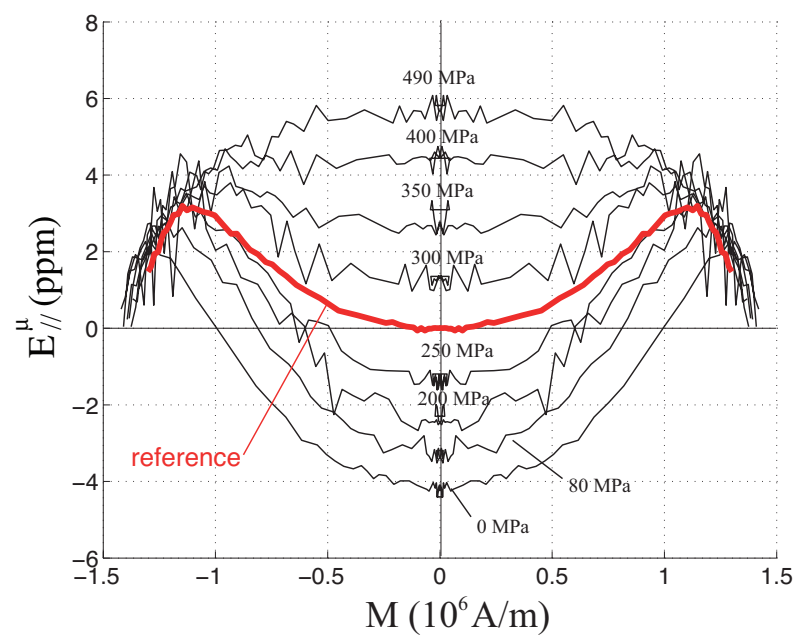

(a) Experiments

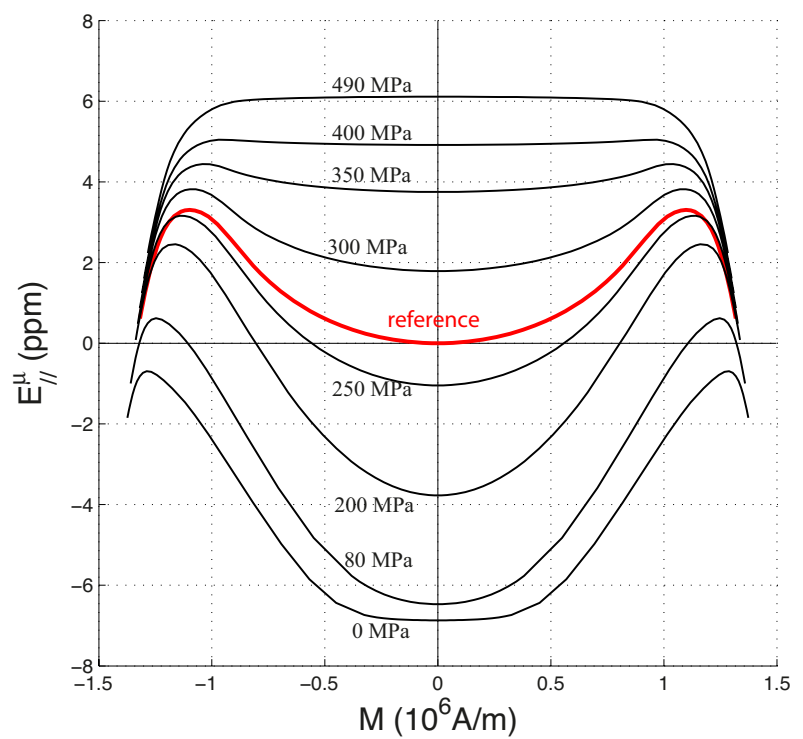

(b) Modeling

Figure 29: Anhysteretic longitudinal magnetostrictive behavior of the sample prestrained at $1 \%$ and reloaded at various stress levels. 


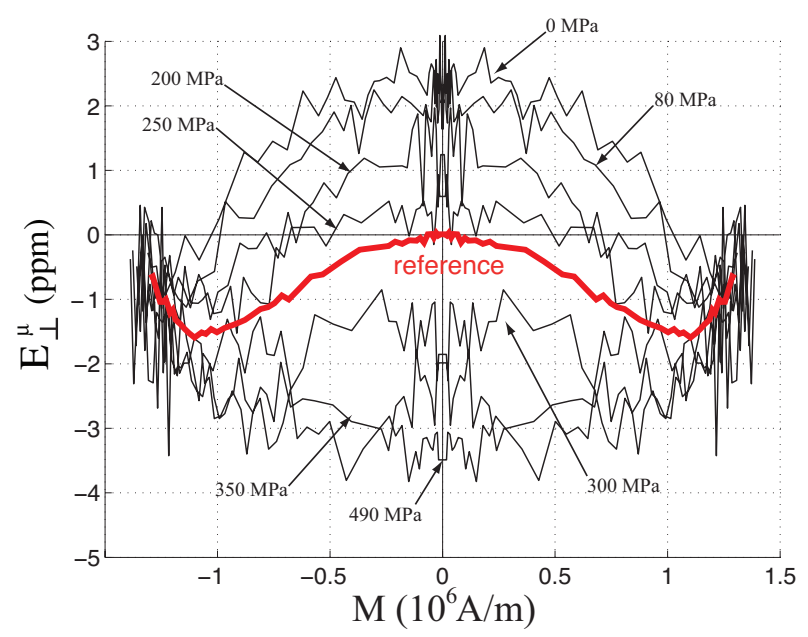

(a) Experiments

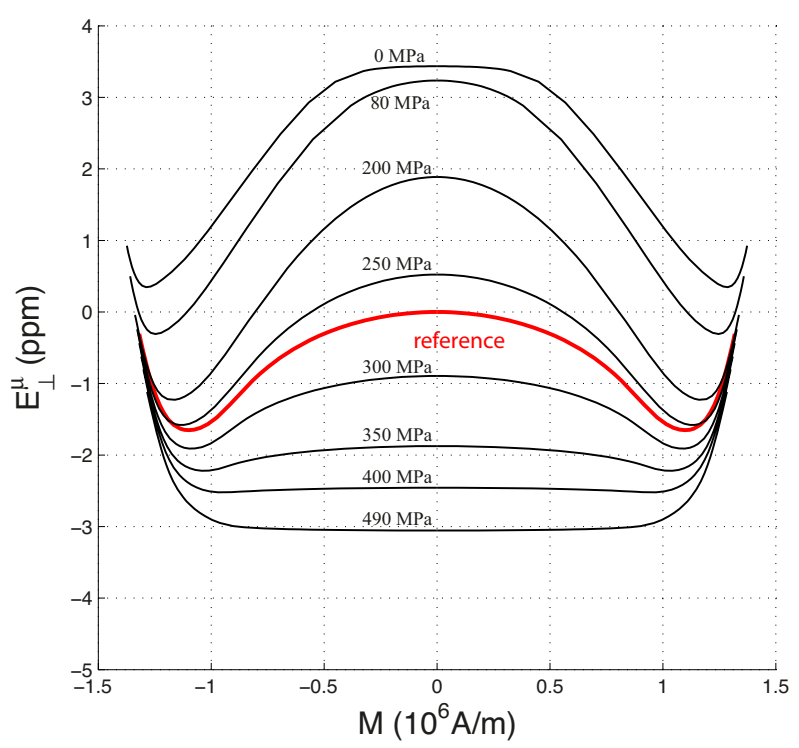

(b) Modeling

Figure 30: Anhysteretic transverse magnetostrictive behavior of the sample prestrained at $1 \%$ and reloaded at various stress levels. 


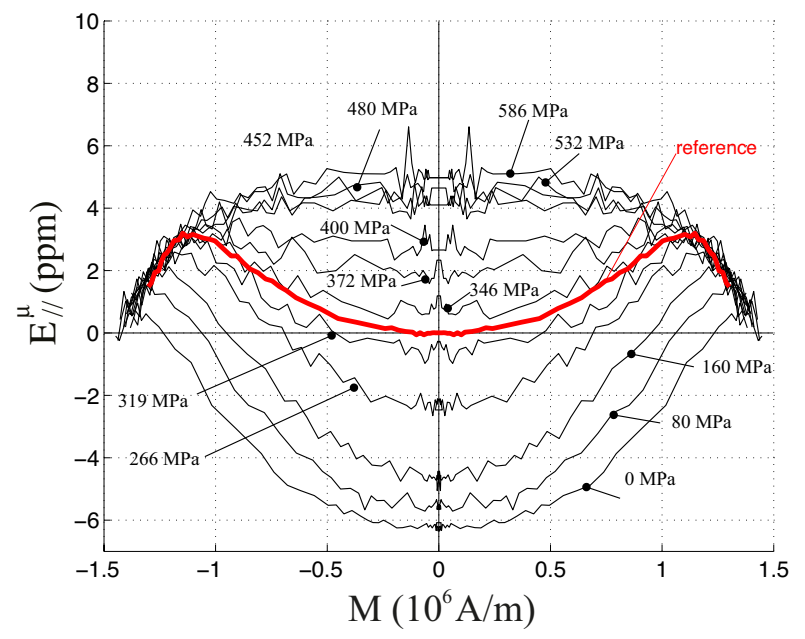

(a) Experiments

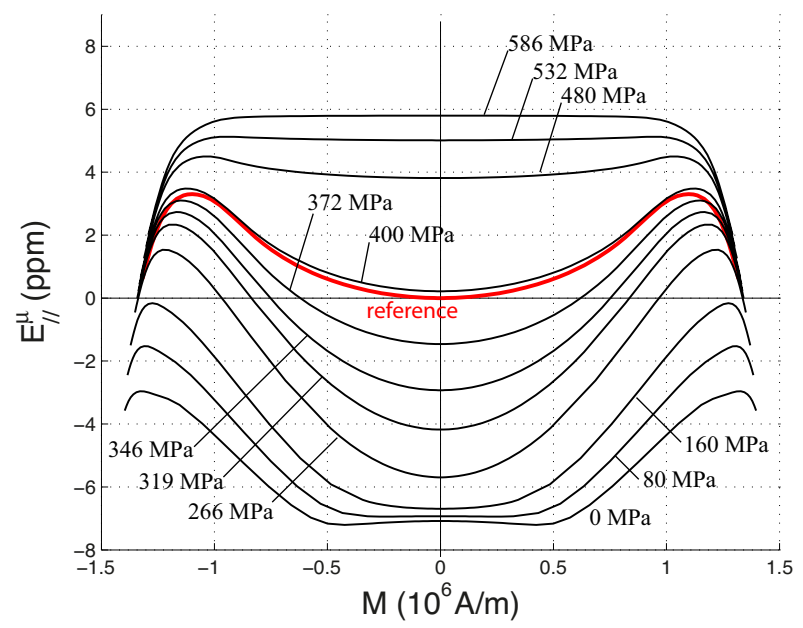

(b) Modeling

Figure 31: Anhysteretic longitudinal magnetostrictive behavior of the sample prestrained at 3\% and reloaded at various stress levels. 


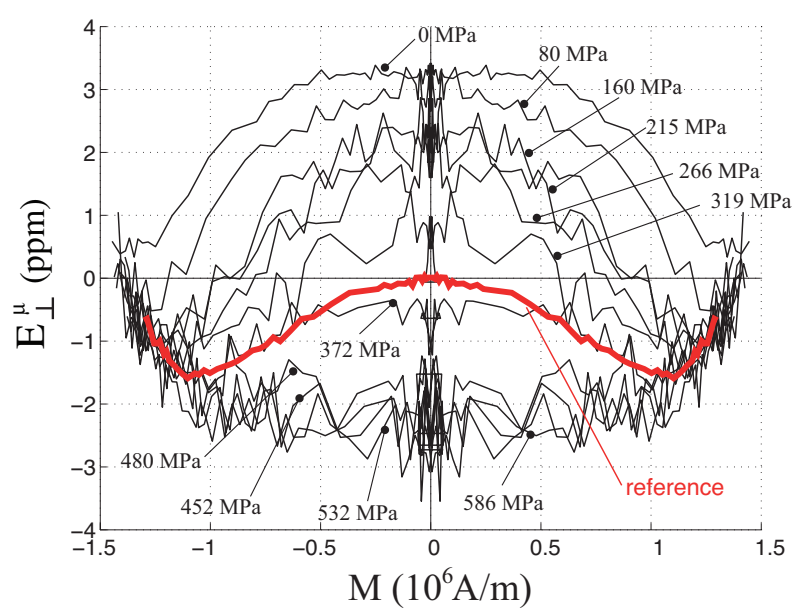

(a) Experiments

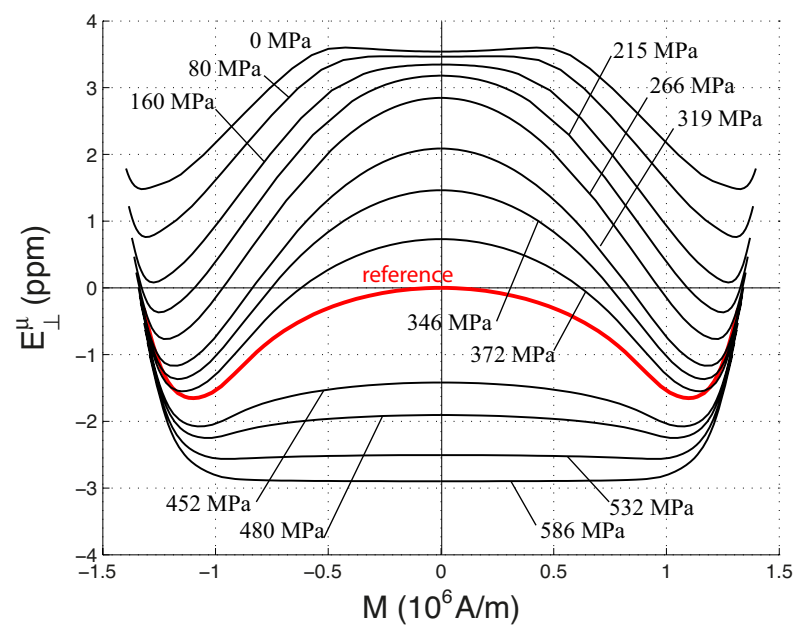

(b) Modeling

Figure 32: Anhysteretic transverse magnetostrictive behavior of the sample prestrained at 3\% and reloaded at various stress levels.

The so-called $\Delta E$ effect $\left(\epsilon_{/ /}^{\mu}(M=0, \Sigma)\right.$ see [61]) has been plotted in Figure 33 for all specimens in order to quantify the effect of the stress reloading and to accurately estimate $\Sigma_{c}^{\mu}$. The magnetostrictive behavior cannot be summarized by this simple parameter $\Delta E$ but allows one to illustrate the major trends. It appears that plastic strain leads to a progressive shift of the curves in the magnetostriction-stress plane comparable to a frame displacement. The magnetostrictive recovery stress $\Sigma_{c}^{\mu}$ is corresponding to the stress value at the intersection between the $E_{/ /}^{\mu}(M=0, \Sigma)$ curves and the $E_{/ /}^{\mu}=0$ axis. Despite some discrepancies in amplitude and recovery stress, the modeling gives results in remarkable agreement with experiments. This result again proves that the modeling of the plastic strain effect on the behavior of DP780 steel does not require the consideration of any change of the magnetic behavior of the phases but only the 
stress field associated with plasticity.

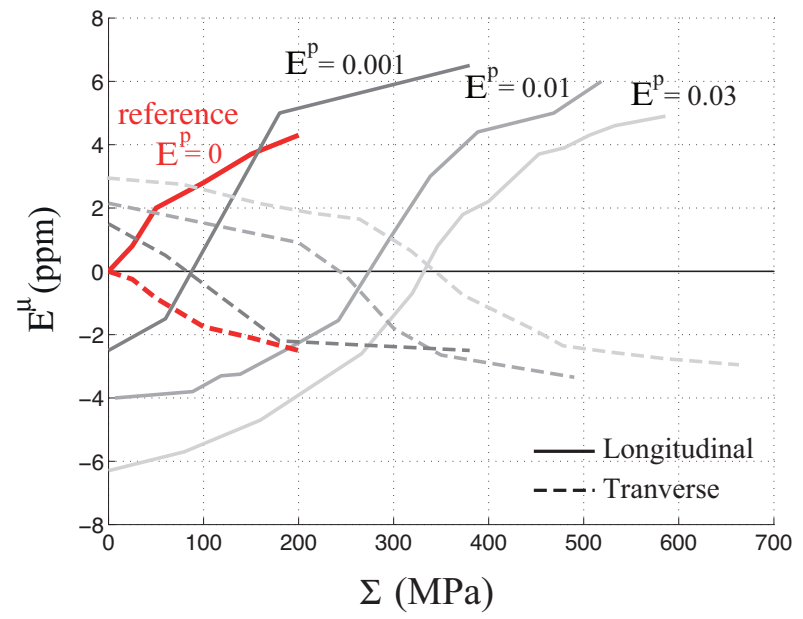

(a) Experiments

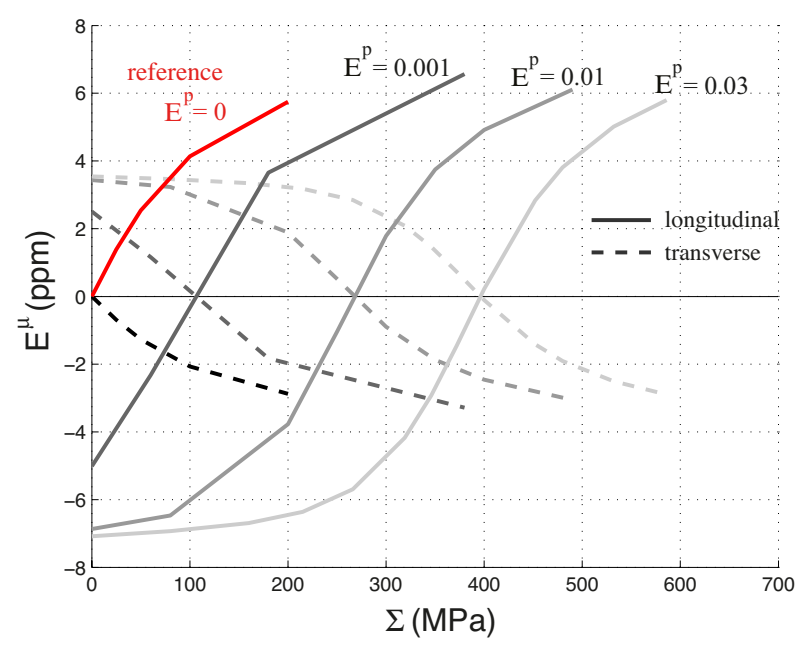

(b) Modeling

Figure 33: Longitudinal and transverse $\Delta E$ effect of the plastic strained samples. 


\section{Discussion}

\subsection{Results analysis, recovery stress}

Comparisons between experiments and modeling lead to very interesting results. The mechanical approach associated to a two-phase magnetomechanical model proved its efficiency to reproduce both magnetization and magnetostriction behaviors of plastic strained DP steel (up to $3 \%$ of plastic strain level). Two major defects have been nonetheless identified: $1 /$ the nonmonotonic variation of the magnetic behavior at high positive stress, not reproduced by the modeling; 2 / a perceptible discrepancy between experimental and modeled recovery stresses.

Concerning the first point, it is possible to introduce in the modeling a demagnetizing stress factor as introduced in [62] for the full multiscale model. This introduction would have increased the number of parameters to be identified without fundamentally changing the learnings of the study. The values reported in Table 5 address the second point. In this table, the experimental magnetic and magnetostrictive recovery stresses $\Sigma_{c}^{\chi}$ and $\Sigma_{c}^{\mu}$ have been reported with estimated evaluation errors. They are compared to the recovery stress evaluated from the modeling results $\Sigma_{c}^{m}$. We observe first that $\Sigma_{c}^{m}$ values correspond precisely to the $\frac{3}{2} X$ values. This result was expected since only the soft phase significantly participates to the magnetic behavior of the material. When $\Sigma_{c}^{m}=\frac{3}{2} X$ the stress in the soft phase is nul. The high tensile stress in the hard phase has only a minor effect. We observe on the other hand that $\Sigma_{c}^{\chi}$ and $\Sigma_{c}^{\mu}$ values are in agreement but slightly different. The recovery stress extracted from the model and evaluated from Cottrell's method gives results close to results issued from the magnetic measurements. Measurement errors are high for the lowest plastic strain level due to a too small number of experimental points. The discrepancy observed at the highest plastic strain level may have different origins. One first source of discrepancy is associated with the Cottrell's method itself. The second source of discrepancy is the imperfect modeling of the martensite phase. Indeed $K_{1}, M_{s}, \lambda_{100}$ and $\lambda_{111}$ look more like fitting parameters than physical parameters. Magnetoelastic coupling in the martensite phase consequently may be badly modeled. Finally, the main assumption of the model based on the insensitivity of the behavior of each phase to plastic deformation is probably no more valuable at high strain. Indeed, the wavelength characteristic of the internal stresses associated with the plasticity decreases with increasing strain [17]. Initially intergranular (interphase by extension), they become progressively transgranular. The definition of hard and soft phase should be revisited for high strains.

\begin{tabular}{|l|c|c|c|}
\hline$E^{p}$ & $\Sigma_{c}^{\chi}(\mathrm{MPa})$ & $\Sigma_{c}^{\mu}(\mathrm{MPa})$ & $\Sigma_{c}^{m}(\mathrm{MPa})$ \\
\hline \hline 0.001 & $180 \pm 50$ & $80 \pm 20$ & 100 \\
\hline 0.01 & $300 \pm 10$ & $270 \pm 10$ & 270 \\
\hline 0.03 & $370 \pm 10$ & $330 \pm 10$ & 400 \\
\hline
\end{tabular}

Table 5: Recovery stresses - experimentally estimated $\left(\Sigma_{c}^{\chi}, \Sigma_{c}^{\mu}\right)$ with errors and foreseen by the model $\left(\Sigma_{c}^{m}\right)$.

\subsection{Limit of the mechanical description of the plastic strain effect on the magnetic behavior}

Measurements of the hysteresis loops of the plastically deformed material were performed simultaneously with anhysteretic measurements. These complementary measurements provide 
information about other magnetic parameters such as the coercive field, remanent magnetization and losses. This is an important point since loss characteristics are for example critical parameters for electrical steels. On the other hand, the former results concerning the influence of plasticity or reloaded stress usually address this kind of behavior. In this paper, we did not focus on these behaviors because the modeling proposed is an anhysteretic modeling whose validation requires only anhysteretic measurements. Figure 34 shows typical magnetic hysteresis loops obtained at $0.1 \mathrm{~Hz}$ frequency (quasi static condition - same maximum magnetic field $\mathrm{H}_{m}=16 \mathrm{kA} / \mathrm{m}$ ) for the non-deformed and plastic strained samples at unloaded state. As already observed by many authors, plastic straining drastically changes the loops shape: enlargement, rotation, and Villari reversal phenomenon are easily observable.

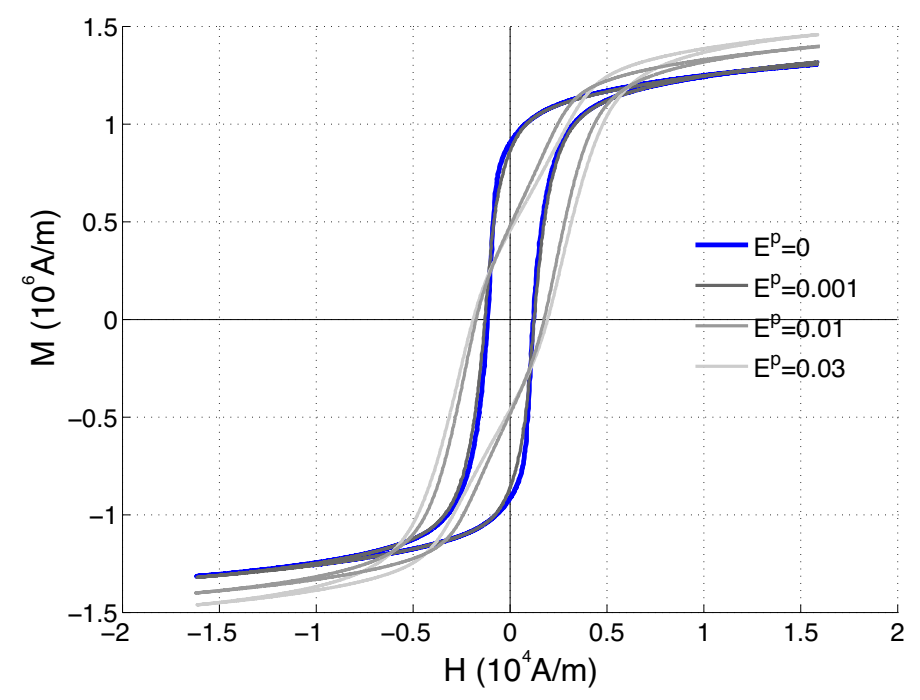

Figure 34: Hysteresis loops of plastified dual-phase steel at different levels without reloaded stress $-\mathrm{f}=0.1 \mathrm{~Hz}$.

Figures 35 shows the effect of reloaded stress on the coercive field $H_{c}^{16000}$ (the magnetic field value extracted from the major loop at $\mathrm{M}=0$ ) of the plastically strained samples. In the unloaded state, plasticity leads to a sharp and strongly non-linear change of the coercive field in accordance with results of literature. We remark first that the applied stress has only a small influence on the coercive field of the unstrained sample. The variation of coercive field of the sample strained at $0.1 \%$ is low and effect of reloaded stress is weak and difficult to analyze due to the too small number of loading states. The reloaded stress allows on the contrary a large recovering of the coercive field of $1 \%$ and $3 \%$ strained sample. This recovery remains nonetheless partially incomplete and occurs at stress levels much larger that the recovery stresses of anhysteretic quantities. This result joins the former results of Iordache obtained on a $3 \% \mathrm{Si}-\mathrm{Fe}$ alloy [24]. Indeed the coercive field is well known to be very defect sensitive. The irreducible part of the coercive field can probably be related to the contribution of these defects. These results show that mechanical description of the plastic strain effect on the magnetic behavior must be complemented by a metallurgical description.

Results presented in this part are surely insufficient for a complete discrimination between effects. They have to be complemented by other experiments. Extension to the hysteresis modeling as proposed in [62] is another important issue. 


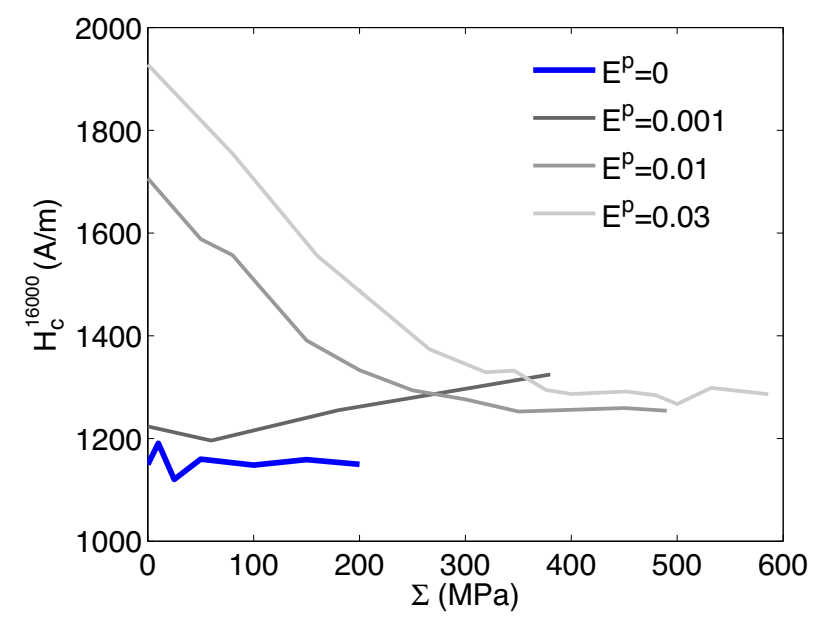

Figure 35: Effect of reloading stress on the coercive field of the plastically strained samples.

\subsection{Modeling of former experimental results}

In this last subsection, we propose a short revisit of some former results in order to improve their interpretation using the two-phase model. Results concern non-oriented $3 \% \mathrm{Si}$-Fe electrical steel plastic strained at different levels and for which a measurement of the kinematic hardening have been made (see $[17,24,27]$ ). The major difference with the present study is that the material is single phased. Consequently we must use complementary informations to define the volume fraction of soft and hard phases. For the illustration, we took the example of $3 \% \mathrm{Si}-\mathrm{Fe}$ strained at $0.5 \%, 3.6 \%$ and $9.8 \%$ whose magnetic characterization is detailed in [17]. Table 6 reports the kinematic hardening associated with each sample and measured using oligocyclic tensioncompression tests. Indeed microstructural observations [64] showed that the kinematic hardening is mainly due to intergranular strain incompatibilities at low strain, with a high influence of the area of grain boundaries. A mix of intergranular and transgranular incompatibilities is observed at intermediate strain and clear high transgranular incompatibilities characterize the stress fields at the highest strain. These observations allow to conclude that the volume fraction of soft phases is dominant comparing to the volume fraction of hard phases (in accordance with Xray measurements of Cullity [2]). As a simplification, we decided to choose the same ratio of phases using $f_{s}=0.9$ and $f_{h}=0.1$ for the modeling whatever the plastic strain level. Table 7 reports the physical constants for iron-silicon. Magnetostrictive and magnetocystalline constants and saturation magnetization reported in the table are very well known for this material. The configuration stress has been chosen at zero for simplification. The value chosen for the initial susceptibility is a standard value.

Figure 36 plots the modeling results of the magnetization behavior. Figure 37 plots the modeling results of longitudinal magnetostriction behavior. Despite the hypothesis of invariance of behavior in each phase and the strong simplification concerning the volume fraction of phases, results are in good qualitative agreement with experiments reported in [17] and [30] for magnetic measurements and in [30] for magnetostrictive measurements. The final experiments vs. modeling comparison concerns the previous results of Cullity [2]. The strong non-linear influence of plasticity is especially highlighted. Figure 38a reports some longitudinal magnetostriction mea- 


\begin{tabular}{|l|c|}
\hline$E^{p}$ & $3 / 2 X(\mathrm{MPa})$ \\
\hline \hline 0.005 & 80 \\
\hline 0.036 & 130 \\
\hline 0.098 & 170 \\
\hline
\end{tabular}

Table 6: Kinematic hardening and volume fraction of soft and hard phases at the three considered plastic strain levels.

\begin{tabular}{cccccc} 
Param. & $M_{s}$ & $K_{1}$ & $\lambda_{100} ; \lambda_{111}$ & $\chi_{0}$ & $\sigma^{\text {conf }}$ \\
\hline \hline Value & $1.61 \times 10^{6}$ & 38 & $23.5 ;-4.5$ & 3000 & 0 \\
Unit & $\mathrm{A} / \mathrm{m}$ & $\mathrm{kJ} . \mathrm{m}^{-3}$ & $\mathrm{ppm}$ & - & $\mathrm{MPa}$
\end{tabular}

Table 7: Parameters of multidomain modeling for 3\% Si-Fe alloy.

surements under applied stress (in ksi) for pure iron. The magnetostriction of a plastic strained sample (3\%) in the unloaded state is also reported (dotted lines). The associated modeling (same stress levels) is plotted in Figure 38b using the parameters of the ferrite phase (Table 3 - except for $\chi_{0}=5000$ and $\sigma^{c o n f}=12 \mathrm{MPa}$ ). Obviously, the volume fraction of the soft and hard phases, as the magnitude of kinematic hardening are not known. The parameters indicated in the figures are chosen close to the parameters used for the iron-silicon alloy. The modeling and experimental results are in very good agreement, despite the global level predicted by the model is about 2 ppm higher than experiments.

These results prove that the modeling approach applied initially to a real bi-phased material can be extended to many other families of magnetic materials thanks to an appropriate choice of volume fraction and via the knowledge of the kinematic hardening. The modeling of former results of Neurath, Makar, Hug, Iordache, Landgraf and many others could be made in the same way.

The modeling of so-called magnetoplastic anisotropy $[17,55]$ not represented here is nonetheless poorly reproduced. Indeed this modeling requires an appropriate description of the effect of high tensile stress on the magnetic behavior by the introduction of a demagnetizing stress tensor [62]. This point will be addressed in a future communication. 


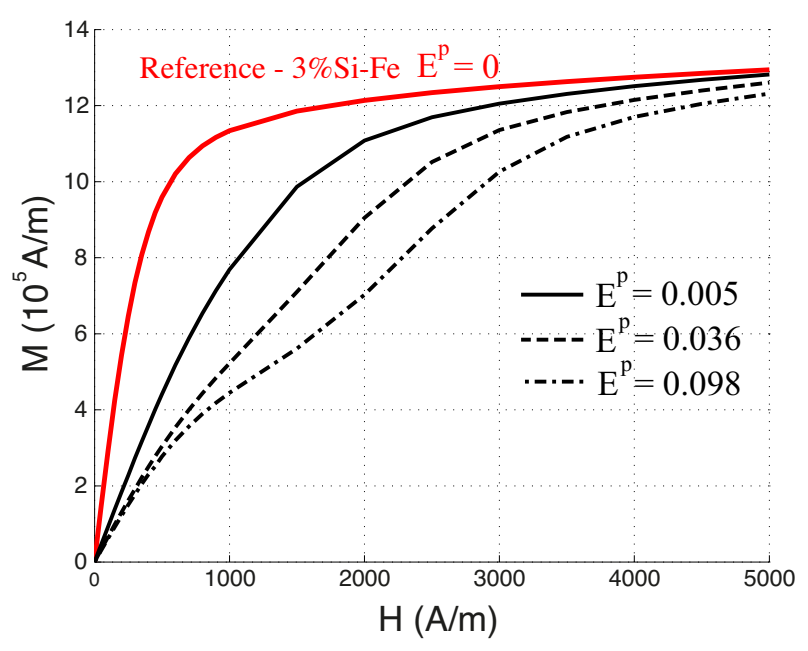

Figure 36: Two-phase modeling of the influence of plasticity on the magnetic behavior of non-oriented 3\% Si-Fe alloy.

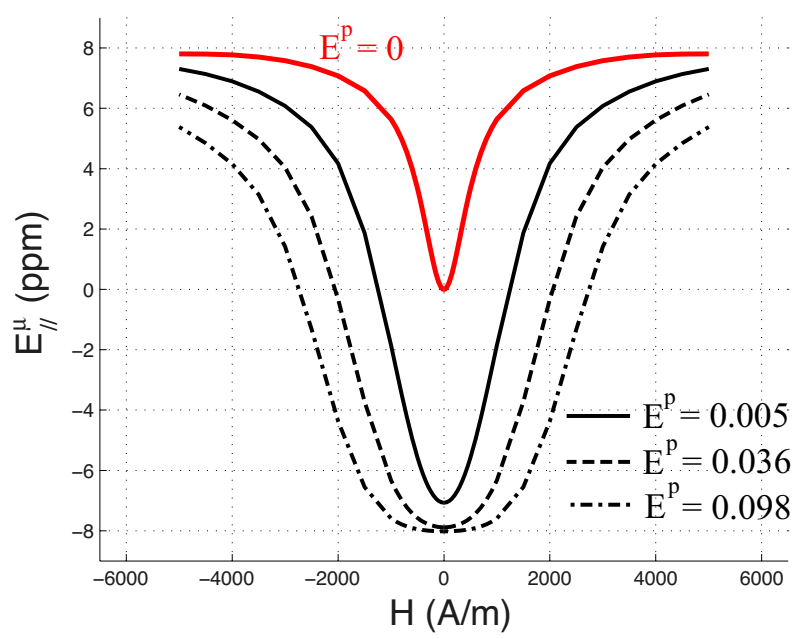

Figure 37: Two-phase modeling of the influence of plasticity on the longitudinal magnetostriction behavior of nonoriented $3 \% \mathrm{Si}-\mathrm{Fe}$ alloy. 


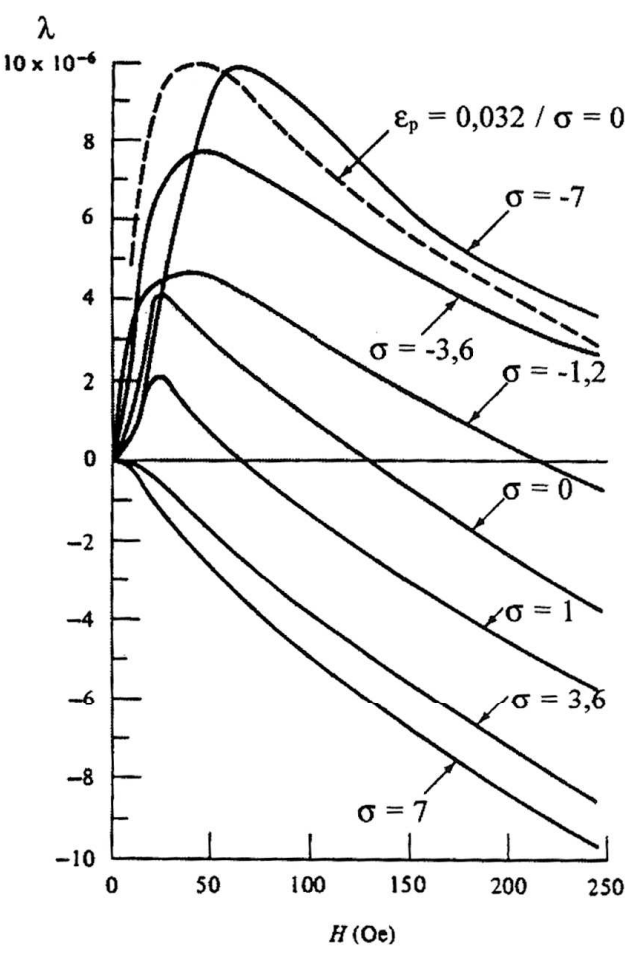

(a) Experiments - see [2]

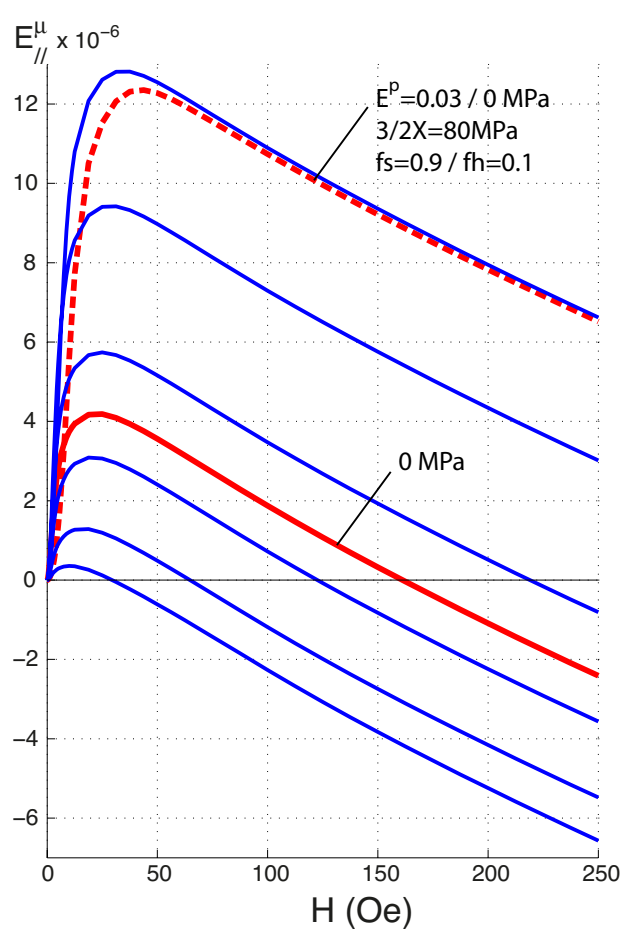

(b) Modeling - same stress levels

Figure 38: Longitudinal magnetostrictive behavior of pure iron - effect of applied stress (in ksi) vs. effect of a 3\% plastic strain in the unloaded state (dotted lines).

\section{Conclusion}

In this paper, a two-phase modeling of the magnetic and magnetostrictive behavior of plastic strained magnetic materials has been proposed. This approach supposes some simple assumptions concerning the behavior of both phases or the phase distribution. It supposes the knowledge of the kinematic strengthening state in the material too, but very few real fitting parameters are required. The physical principle used in this modeling is to consider that plastic strain acts on the magneto-mechanical behavior through its associated internal stresses and the magneto-elastic coupling effect that occurs within the phases. This theoretical approach is complemented by experimental observations of the complete magnetomechanical behavior of dual-phase steel including magnetostriction measurements under applied stress. A hard martensitic second phase leads to a strong kinematic hardening of the material during plasticity. The anhysteretic magnetic measurements allow the estimation of some peculiar uniaxial stress levels able to recover the magnetic and magnetostrictive behavior (going back to the initial properties of the material). These stress levels are found to be close to the stress associated with the position of the actual yield surface centre, thanks to the very low participation of the martensite in the average magnetic behavior. It has to be noticed that the pinning effect usually associated with plastic strain has not been considered in this modeling, explaining why the coercive field variations are insufficiently described. Extension of the modeling to hysteretic behavior is nonetheless possible 
following the principles developed in [62].

On the other hand it has been shown how this approach can be extended to more classical materials like mild steels, electrical steels and other single phase materials, submitted to plastic strain. This work offers a new and simple way to model the effect of plasticity (including multiaxial plasticity [52]) on this wide range of materials.

Finally this work opens an interesting way to estimate the mechanical state in a deformed material. Indeed a clear relationship has been made between kinematic hardening and magnetic behavior in case of a uniaxial tensile test. An interesting feature would be to apply this new approach to the measurement of the kinematic hardening during a multiaxial testing. It gives some insight of future magnetic non-destructive monitoring techniques.

\section{Acknowledgements}

The authors wish to thank ArcelorMittal for providing the material, F.S. Mballa Mballa for microstructural observations and complementary measurements, and M. Callahan for its careful reading. This work was partly funded by the French Agency for National Research (ANR) under contract MATETPRO08-322447.

\section{References}

[1] R.M. Bozorth, Ferromagnetism, Noth Vorstand, 1951.

[2] B.D. Cullity, Introduction to magnetic Materials, Addison-Wesley Publishing Company, 1972.

[3] D.C. Jiles, NDT International, 21 (1988), pp.311-319.

[4] G. Dobmann. Electromagnetic Nondestructive Evaluation (VII). IOS Press, 2006.

[5] M. Augustyniak, Z. Usarek, J. Non destruct. Eval, 34:21 (2015), pp.1-9.

[6] G. Dobmann, Physical basics and industrial applications of 3MA micromagnetic multiparameter microstructure and stress analysis. 12th Int. Workshop on Electromagnetic NDE (ENDE2007), Cardiff, UK, 2007.

[7] Y. Gabi, A. Kedous-Lebouc, G. Meunier, B. Wolter, O. Geoffroy, P. Meilland, P. Labie, C. Guérin and O. Martins, Proc. 19th Conf. Comput. Electromagn. Fields, (2013) pp.48-49.

[8] Y. Gabi, B. Wolter, A. Gerbershagen, M. Ewen, P. Braun, O. Martins, IEEE Trans. Mag., 50, 4, (2014), 7026504

[9] F.S. Mballa-Mballa, O. Hubert, S. Lazreg, P. Meilland, "Multidomain modelling of the magneto-mechanical behaviour of dual-phase steels". 18th WCNDT - World Conference on Nondestructive Testing. Durban (South Africa) 2012.

[10] A.V. Marmulev, L.M. Kaputkina, E.I. Poliak, J.M. Turon, Machines, Technologies, Materials, 9 (2013), pp.27-29.

[11] G. Vértesy, I. Mészáros, I. Tomás̃c, NDT \& E International, 54, (2013), pp.107-114

[12] M. Radu, J. Valy, A.F. Gourgues, F. Le Strat, A. Pineau, Scripta Materialia, 52 (2005), pp.525-530.

[13] L. Zhao, N. H. van Dijk, A. J. Lefering, J. Sietsma, Journal of Materials Science, 484 (2013), pp.1474-1479.

[14] J. Degauque, B. Astié, L.P. Kubin, Phys. Stat. Sol. (a) 45 (1978) pp.493-501.

[15] H.R. Hilzinger, H. Kronmüller, Phys. Lett. 51A (1975), pp.59-60.

[16] E. Hug, O. Hubert, M. Clavel, IEEE Trans. Mag., 1 (1997), pp.763-771.

[17] O. Hubert, M. Clavel, I. Guillot, E. Hug, Jal de Physique IV. 9 (1999), pp.207-216.

[18] D. C. Jiles, J. Phys. D: Appl. Phys. 21 (1988), pp.1196-1204.

[19] L. J. Swartzendruber, G. E. Hicho, H. D. Chopra, S. D. Leigh and G. Adam, J. Appl. Phys. 81, (1997) pp.4263-4265.

[20] J.M. Makar, B.K. Tanner, J. Magn. Magn. Mater. 184 (1998) pp.193-208.

[21] J.M. Makar, B.K. Tanner, J. Magn. Magn. Mater. 187 (1998) pp.353-365.

[22] J.M. Makar, B.K. Tanner, NDT\&E International, 312 (1998) pp.117-127.

[23] O. Perevertov, J. Phys. D - Appl. Phys., 40 (2007) pp.949-954.

[24] V.E. Iordache, E. Hug and N. Buiron, Mater. Sci. Eng. A. 359, (2003) pp.62-74.

[25] O. Hubert, N.Jendly and L. Daniel, Steel Res. Int., 766 (2005), pp.440-447.

[26] E. Hug, O. Hubert, I. Guillot, J. Magn. Magn. Mater. 215-216 (2000) pp.197-200.

[27] E. Hug, et al., Mat. Sc. and Eng. A, A332, (2002), pp.193-202.

[28] P. Baudouin, Y. Houbaert, S. Tumanski, J. Magn. Magn. Mat., 254-255 (2003), pp.32-35.

[29] M. J. Sablik, S. Rios, F. J. G. Landgraf, T. Yonamine and M. F. de Campos, J. Appl. Physics, 97 (2005) $10 \mathrm{E} 518$. 
[30] O. Hubert, L. Daniel, J. of Magn. Magn .Mater., 304, (2006), p.e489-e491.

[31] L. Piotrowski, M. Chmielewski, B. Augustyniak, J. Magn. Magn. Mater. 410 (2016), pp.34-40

[32] A. Seeger, H. Kronmüller, H. Rieger, H. Traüble, J. Appl. Phys. 35 (1964), pp.740-748.

[33] H.R. Hilzinger, H. Kronmüller, J. Magn. Magnetic Mat. 2, (1976), pp.11-17.

[34] O. Hubert and E. Hug, Mat. Science \& Techno., 11 (1995), pp.482-487.

[35] F. Ossart, E. Hug, O. Hubert, C. Buvat, R. Billardon, IEEE Trans. Magn. 36 (2000) pp.3137-3140.

[36] M. Hofmann, H. Naumoski, U. Herr, and H.G. Herzog, IEEE Trans. Mag., 52, 2, (2016), 2000114.

[37] A. H. Qureshi, L. N. Chaudhary, J. Appl. Phys., 41 (1970), pp.1042-1043.

[38] J.M. Makar, B.K. Tanner, J. of Magn. Magn .Mater., 222 (2000) pp.291-304.

[39] M. J. Sablik, J. Appl. Physics, 97 (2001), pp.5610-5613.

[40] B. Astié, J. Degauque, J.L. Porteseil and R. Vergne, J. of Magn. Magn. Mater., 28, (1982) pp.149-153.

[41] J. Degauque, Mem. et Etudes Scient. Rev. Mtal. 1 (1985), pp.5-23.

[42] F.J.G. Landgraf, M. Emura, J. of Magn. Magn. Mater., 242-245, (2002) pp.152-156.

[43] L. Daniel, O. Hubert, N. Buiron, R. Billardon, J. Mech. Phys. Sol., 56 (2008), pp.1018-1042.

[44] O. Hubert, L. Daniel, J. of Magn. Magn. Mater. 320 (2008), pp.1412-1422.

[45] O. Hubert, L. Daniel, J. of Magn. Magn. Mater., 323, (2011) pp.1766-1781.

[46] C. Bormio-Nunes, O. Hubert, J. of Magn. Magn .Mater., 393 (2015), pp.404-418.

[47] J. Lemaitre, J.L. Chaboche Mechanics of solid materials, Cambridge University Press, 1994.

[48] X. Feaugas, Acta Mater. 47 (1999) pp.3617-3632.

[49] H. Mughrabi, Acta Metallurgica, 9 (1983), pp.1367-1379.

[50] V. E. Iordache, F. Ossart, E. Hug, J. Magn. Magn. Mater. 254-255 (2003), pp.57-59.

[51] R.A. Langman, NDT international, (1981) pp.255-262.

[52] M. Küpferling, F. Fiorillo, V. Basso, G. Bertotti and P. Meilland, J. of Magn. Magn .Mater., 320 20 (2008) pp.527530.

[53] R. Hill, J. of the Mech. Phys. Solids 13 (1965) pp.89-101.

[54] M. Berveiller, A. Zaoui, J. Mech. Phys. Solids, no 5-6 26 (1978) pp.325-344.

[55] O. Hubert, L. Hirsinger, E. Hug, J. of Magn. Magn. Mater., 196-197(1999) pp.322-324.

[56] S. Lazreg, O. Hubert, J. Appl. Phys., 109, (2011) 07E508.

[57] L. Daniel, N. Galopin, European Physical Journal - Applied Physics, 42 (2008), pp.153-159.

[58] S. Allain and O. Bouaziz, Mat. Sci. \& Eng.A, 496 (2008), pp.329-336.

[59] G. V. Bida, A. P. Nichipuruk, T. P. Tsar'kova, Russian Journal of Nondestructive Testing, 37, Issue 2, (2001), pp.79-99.

[60] O. Hubert, L. Daniel, R. Billardon, J. of Magn. Magn .Mater., 254-255 (2003) pp.352-354.

[61] L. Daniel, O. Hubert, Eur. Phys. J. Appl. Phys. 45 (2009) 31101.

[62] L. Daniel, M. Rekik, O. Hubert, Arch. of Appl. Mech., 84, Issue 9 (2014), pp.1307-1323.

[63] O. Hubert, M. Chaabane, J. Jumel, V. Maurel, F. Alves, A. Bensalah, M. Besbes, K. Azoum, F. Bouillault, Przeglad Elektrotecniczny, R81, Issue 5 (2005), pp.19-23.,

[64] O. Hubert, E. Hug, I. Guillot and M. Clavel, J. Physique IV, 8, 6 (1998) pp.515-518. 